\title{
Numerical null controllability of the heat equation through a least squares and variational approach
}

\author{
ARNAUD MÜNCH${ }^{1}$ and PABLO PEDREGAL ${ }^{2}$ \\ ${ }^{1}$ Laboratoire de Mathématiques, Université Blaise Pascal (Clermont-Ferrand 2), UMR CNRS 6620, \\ Campus des Cézeaux, 63177 Aubière, France \\ email: arnaud.munch@math.univ-bpclermont.fr \\ ${ }^{2}$ E.T.S. Ingenieros Industriales, Universidad de Castilla La Mancha, Campus de Ciudad Real, Spain \\ email: pablo.pedregal@uclm.es
}

(Received 26 April 2013; revised 13 January 2014; accepted 15 January 2014;

first published online 13 February 2014)

This work is concerned with the numerical computation of null controls for the heat equation. The goal is to compute an approximation of controls that drives the solution from a prescribed initial state at $t=0$ to zero at $t=T$. In spite of the diffusion of the heat equation, recent developments indicate that this issue is difficult and still largely open. Most of the existing literature, concerned with controls of minimal $L^{2}$-norm, make use of dual convex arguments and introduce backward adjoint system. In practice, the null control problem is then reduced to the minimization of a dual conjugate function with respect to the final condition of the adjoint state. As a consequence of the highly regularizing property of the heat kernel, this final condition - which may be seen as the Lagrange multiplier for the null controllability condition does not belong to $L^{2}$, but to a much larger space than can hardly be approximated by finite (discrete) dimensional basis. This phenomenon, unavoidable whatever be the numerical approximation used, strongly deteriorates the efficiency of minimization algorithms. In this work, we do not use duality arguments and in particular do not introduce any backward heat equation. For the boundary case, the approach consists first in introducing a class of functions satisfying a priori the boundary conditions in space and time, in particular the null controllability condition at time $T$, and then finding among this class one element satisfying the heat equation. This second step is done by minimizing a convex functional among the admissible corrector functions of the heat equation. The inner case is performed in a similar way. We present the (variational) approach, discuss the main features of it and then describe some numerical experiments highlighting the interest of the method. The method holds in any dimension but, for the sake of simplicity, we provide details in the one-space dimensional case.

Key words: Heat equation; Null controllability; Numerical approximation; Variational approach

\section{Introduction}

We are concerned in this work with the null controllability problem for the 1-dimensional (1D) heat equation for both the boundary and the inner case. We denote by $T$ any strictly positive real, $\omega$ any non-empty (small) subset of $(0,1)$ and $1_{\omega}$ the characteristic function 
of $\omega$. We introduce the diffusion function $a$ assumed to be uniformly bounded and strictly positive all over the interval $(0,1)$ :

$$
a \in C^{1}([0,1]), \quad a(x) \geqslant a_{0}>0 \quad \forall x \in[0,1] .
$$

We also introduce in the sequel the notation

$$
q_{T}=\omega \times(0, T), \quad Q_{T}=(0,1) \times(0, T), \quad \Sigma_{T} \in\{1\} \times(0, T) .
$$

The boundary control problem that we consider here can be stated as follows (see $[5,22])$ : Given any initial data, $u_{0} \in L^{2}(0,1)$, find a control function $w \in L^{2}\left(\Sigma_{T}\right)$ such that the unique (weak) solution $u \in C^{0}\left([0, T] ; H^{-1}(0,1)\right) \cap L^{2}\left(0, T ; L^{2}(0,1)\right)$ of the homogeneous linear equation

$$
\left\{\begin{array}{lc}
u_{t}-\left(a(x) u_{x}\right)_{x}=0 & (x, t) \in Q_{T}, \\
u(x, 0)=u_{0}(x) & x \in(0,1), \\
u(0, t)=0, u(1, t)=w(t) & t \in(0, T)
\end{array}\right.
$$

satisfies the null controllability condition

$$
u(\cdot, T)=0 \quad \text { in } \quad(0,1)
$$

As it is usual, the solution is defined by transposition. Similarly, the inner (or distributed) control problem may be stated as follows: Given any initial data $u_{0} \in L^{2}(0,1)$, find a control function $f \in L^{2}\left(q_{T}\right)$ such that the unique solution $u \in C^{0}\left([0, T] ; L^{2}(0,1)\right) \cap$ $L^{2}\left(0, T ; H_{0}^{1}(0,1)\right)$ of the homogeneous linear equation

$$
\left\{\begin{array}{lc}
u_{t}-\left(a(x) u_{x}\right)_{x}=f 1_{\omega} & (x, t) \in Q_{T}, \\
u(0, x)=u_{0}(x) & x \in(0,1), \\
u(t, 0)=u(t, 1)=0 & t \in(0, T)
\end{array}\right.
$$

satisfies (1.4).

In the 1D space case, these controllability problems are known to be solvable since the 1970s: We refer to the earlier contributions [8,32] for some proofs based on spectral arguments. For more recent and general results based on duality arguments and the Carleman-type estimates, we refer to $[1,14,23]$. As is usual in these types of problems, the dual approach allows to reduce the controllability problem to a suitable observability result for the adjoint system. Moreover, in the spirit of the celebrated Hilbert Uniqueness Method introduced by Lions [25], they lead to a practical way of computing controls of a given minimal Sobolev norm.

In order to highlight the underlying difficulties that motivate the search of new methods, let us consider the inner case, which is simpler in many ways with respect to its boundary counterpart. Since there are controls $f \in L^{2}\left(q_{T}\right)$ for (1.5), it is natural to look for the one with minimal $L^{2}$-norm, that is, one seeks to minimize the quadratic functional $J(v)=\frac{1}{2}\|v\|_{L^{2}\left(q_{T}\right)}^{2}$ over the non-empty set

$$
\mathscr{C}\left(u_{0}, T\right)=\left\{(u, f): f \in L^{2}\left(q_{T}\right), u \text { solves }(1.5) \text { and satisfies }(1.4)\right\} .
$$


Since it is difficult to construct pairs in $\mathscr{C}\left(u_{0}, T\right)$ (and a fortiori minimizing sequences !), one may use, following [4], duality arguments to replace the constrained minimization of $J$ by the unconstrained minimization of its conjugate function $J^{\star}$ defined as

$$
J^{\star}\left(\varphi_{T}\right)=\frac{1}{2} \iint_{q_{T}}|\varphi|^{2} d x d t+\int_{0}^{1} u_{0}(x) \varphi(x, 0) d x
$$

over $\varphi_{T} \in \mathscr{H}$ (that will be made precise below), where $\varphi$ is the adjoint backward state associated with (1.5) such that $\varphi(\cdot, T)=\varphi_{T}$. The existence of a positive constant $C=$ $C(\omega, T)$ (the so-called observability constant) such that $C(\omega, T)\|\varphi(\cdot, 0)\|_{L^{2}(0,1)}^{2} \leqslant\|\varphi\|_{L^{2}\left(q_{T}\right)}^{2}$ for all $\varphi_{T} \in L^{2}(0,1)$ implies that $J^{\star}$ is coercive on the Hilbert space $\mathscr{H}$ defined as the completion of $\mathscr{D}(0,1)$ for the norm $\|\varphi\|_{L^{2}\left(q_{T}\right)}$. The control $f$ of minimal $L^{2}\left(q_{T}\right)$-norm is then given by $f=\hat{\varphi} 1_{\omega}$, where $\hat{\varphi}$ is associated with the unique minimizer $\hat{\varphi}_{T}$ in $\mathscr{H}$ of $J^{\star}$ (see $[5,16])$. The difficulty when one wants to approximate such control, that is when one likes to minimize $J^{\star}$ numerically, is that the space $\mathscr{H}$ is huge, in particular, contains $H^{-s}$ for every $s \in \mathbb{N}$, and even elements that may not be distributions. Numerical experiments do suggest that the minimizer $\hat{\varphi}_{T}$ is very singular (we refer to [4] and also to [2,26,27,29] for more details). Note that this phenomenon is independent of the choice of $J$, but is related to the use of dual variables. As we stressed in the Abstract, the equality (1.4) can be viewed as an equality in a very small space (due to the strong regularization effect of the heat kernel). Accordingly, the associated multiplier $\varphi_{T}$ must belong to a large dual space, much larger than $L^{2}(0,1)$, that cannot be represented numerically. We refer to [11], generalizing [4] for weighted-norms, where the same ill-posedness is shown and to [18], where the Tikhonov regularization is introduced and analysed. For these reasons, robust numerical approximations of null controls for parabolic systems remain a challenge.

Recently, an alternative way of looking at these problems and avoiding the introduction of dual variables has been introduced in [30]. It is based on the following simple strategy. Instead of working all the time with solutions of the underlying state equation, and looking for one that may comply with the final desired state, one considers a suitable class of functions complying with required initial, boundary and final conditions, and seeks one of those that is a solution of the state equation. This is in practice accomplished by setting up an error functional defined for all feasible functions, and measuring how far those are from being a solution of the underlying state equation. The task of showing that a problem is controllable amounts to proving that the infimum of the error is a minimum (there is a global minimizer of the error), and that it vanishes. This job requires some interesting analysis as the error functional is not a local, classical integral functional but rather a non-local functional as the one considered for optimal control for distributed parameter systems [24]. Once we have a feasible function with zero error, the control is obtained as the trace of this optimal function (or some other function determined in a unique way through it) in the set where we are entitled to act on the system.

One main practical advantage of this variational approach is that the way to get closer to a solution of the problem is by minimizing a functional that cannot get stuck on local minima because the only critical points of the error turn out to be global minimizers with zero error (see next section). Therefore, a general strategy for numerical approximation consists in using a typical descent algorithm for this error functional. Exploring this 
possibility for the problems described above is the main purpose for this paper. It is organised as follows. In Section 2, first we describe (in a non-technical way) the main ingredients of the variational approach for the heat equation for the boundary case, following [30]. Then we show that [30] may be adapted to address the inner situation. By a general-purpose lemma, we prove that the numerical schemes based on the steepest descent strategy for the error functional always produce numerical sequences that do converge strongly in the appropriate space (3). We then move on to provide the details for the numerical approach based on the Polak-Ribière version of the conjugate gradient $(\mathrm{CG})$ algorithm to minimize the error functional. Section 5 presents several experiments and discuss the practical interest of the approach. Section 6 treats a typical non-linear example to stress the flexibility of the approach. The final section provides a simple method to reduce the cost of controls.

To our knowledge, very few contributions on this topic have appeared since the seminal paper of Carthel-Glowinski-Lions [4] devoted to approximate controllability using duality. This is due to the intrinsic ill-posedness of the problem we have just pointed out. For the null boundary case in $1 \mathrm{D}$ space, we mention the motion planning method introduced in [20] allowing a semi-explicit expression of controlled solutions in term of Gevrey series. This approach has been adapted and numerically developed recently in [29] to obtain inner controls. The recent work [12] - following [14] - extend [4] with the Carleman weighted $L^{2}$-norm, while [11] provides a different variational approach, based on Carleman inequalities, that does not make use of duality argument. For a numerical analysis viewpoint, we also indicate contributions $[3,7,11,19]$.

\section{The least squares variational approach of the null controllability}

We are going to describe in this section the basic ingredients of the variational approach in order to apply it to both boundary and inner controllability problems for the $1 \mathrm{D}$ heat equation.

\subsection{Boundary controllability}

Consider first the boundary controllability problem for the heat equation which consists in finding a function $w \in L^{2}\left(\Sigma_{T}\right)$, such that the solution of the problem (1.3) will comply with $u(x, T)=0$ in $(0,1)$, so that the state $u$ with initial distribution given by initial data $u_{0}$ is led to state 0 at time $T$ under the action of the boundary control $w$ at the right-end point $x=1$. The data $u_{0}$ are given $a$ priori, and the function $a$ is assumed to be uniformly bounded and strictly positive all over the interval $(0,1)$.

The main idea of the variational method, as introduced in [30], consists in setting up an error functional that measures the deviation of functions from being a solution of the underlying heat equation, and minimizing such error over the class of feasible functions that comply with initial, boundary and final conditions. Namely, consider the class of functions

$$
\mathscr{A}=\left\{u \in H^{1}\left(Q_{T}\right) ; u(x, 0)=u_{0}(x), u(x, T)=0, x \in(0,1), u(0, t)=0, t \in(0, T)\right\}
$$


assumed non-empty. This requirement simply demands some compatibility with the vanishing boundary data for $x=0$, precisely that $u_{0}(0)=0$ and that $u_{0}$, as the trace of an $H^{1}$ function over $Q_{T}$, be slightly more regular than $L^{2}(0,1)$, that is, $u_{0} \in H^{1 / 2}(0,1)$. According to the regularising effect of the heat kernel, this assumption may be removed if we assume that the control is zero at time $t=0$. For any $u \in \mathscr{A}$, we define its corrector $v$ over $Q_{T}$ as the solution of the (elliptic) problem

$$
\left\{\begin{array}{lc}
u_{t}-v_{t t}-\left(a(x)\left(u_{x}+v_{x}\right)\right)_{x}=0, & (x, t) \in Q_{T}, \\
v_{t}(x, 0)=v_{t}(x, T)=0, & x \in(0,1), \\
v(0, t)=v(1, t)=0, & t \in(0, T) .
\end{array}\right.
$$

Note that the unique solution of this problem is the minimizer over $H_{0, x}^{1}\left(Q_{T}\right)=\{v \in$ $H^{1}\left(Q_{T}\right), v=0$ on $\left.\{0,1\} \times(0, T)\right\}$ of the regular quadratic functional

$$
\frac{1}{2} \iint_{Q_{T}}\left(\left(\left|v_{t}\right|^{2}+a(x)\left|v_{x}\right|^{2}\right)+u_{t} v+a(x) u_{x} v_{x}\right) d x d t .
$$

The Neumann conditions on the part of the boundary for $t=0$, and $t=T$, are the natural boundary one. One may also consider Dirichlet conditions. Note how this variational problem determining the corrector $v$ is a well-defined problem if $u \in \mathscr{A}$. Even though the corrector function $v$ was introduced in [30] for each time slice $t$ to preserve as general a framework as possible, from the point of view of numerical approximation it is advantageous to define such error function globally in the whole time-space domain $Q_{T}$ by introducing the additional term $-v_{t t}$. This has a regularizing effect on the time-dependence, which is very convenient for numerics.

The error functional is then

$$
E: \mathscr{A} \rightarrow \mathbb{R}^{+}, \quad E(u)=\frac{1}{2} \iint_{Q_{T}}\left(\left|v_{t}\right|^{2}+a(x)\left|v_{x}\right|^{2}\right) d x d t,
$$

where $v$ is the corrector associated with $u$. It turns out that our problem is controllable if and only if the minimum of the error vanishes. In particular, it is well known that the heat problem considered here is controllable, and so the infimum of this error functional is a minimum, and it does vanish [14].

Once we know that the infimum $m \geqslant 0$ is a minimum, we turn to optimality. We define, in a classical way, the variation of $E$ in the direction $U \in \mathscr{A}_{0}$,

$$
<E^{\prime}(u), U>=\lim _{t \rightarrow 0} \frac{E(u+t U)-E(u)}{t},
$$

where the set $\mathscr{A}_{0}$ of admissible variations of $u$ is taken to be

$$
\mathscr{A}_{0}=\left\{U \in H^{1}\left(Q_{T}\right): U(x, 0)=U(x, T)=0, x \in(0,1), U(0, t)=0, t \in(0, T)\right\} .
$$

We easily obtain that

$$
<E^{\prime}(u), U>=\iint_{Q_{T}}\left(v_{t} V_{t}+a(x) v_{x} V_{x}\right) d x d t
$$


where $V \in H_{0, x}^{1}\left(Q_{T}\right)$ is the corrector function associated with $U \in \mathscr{A}_{0}$, that is, the solution of

$$
\left\{\begin{array}{lc}
U_{t}-V_{t t}-\left(a(x)\left(U_{x}+V_{x}\right)\right)_{x}=0, & (x, t) \in Q_{T}, \\
V_{t}(x, 0)=V_{t}(x, T)=0, & x \in(0,1), \\
V(0,1)=V(1, t)=0, & t \in(0, T) .
\end{array}\right.
$$

Multiplying the state equation (2.6) by $v$, integrating by parts and taking into account the boundary conditions on $v$ and $U$, we transform (2.5) into

$$
<E^{\prime}(u), U>=-\iint_{Q_{T}}\left(U_{t} v+a(x) U_{x} v_{x}\right) d x d t, \quad \forall U \in \mathscr{A}_{0} .
$$

Now let us assume that $u \in \mathscr{A}$ is a minimizer for $E$ so that $\left\langle E^{\prime}(u), U\right\rangle=0$ for all $U \in \mathscr{A}_{0}$. This equality implies that $v$ satisfies the backward heat equation

$$
\left\{\begin{array}{lc}
-v_{t}-\left(a(x) v_{x}\right)_{x}=0, & (x, t) \in Q_{T}, \\
a(1) v_{x}(t, 1)=0, & t \in(0, T),
\end{array}\right.
$$

in addition to the boundary conditions

$$
\begin{cases}v_{t}(x, 0)=v_{t}(x, T)=0, & x \in(0,1) \\ v(0, t)=v(1, t)=0, & t \in(0, T)\end{cases}
$$

For any positive time $T>0$, this implies, by the unique continuation property, that the corrector $v$ of $u$ is zero, that $m=E(u)=0$, and the corresponding minimizer $u$ satisfies an homogeneous heat equation. Since $u$ belongs to $\mathscr{A}$, the minimizer of $E$ is then a controlled solution of the heat equation. As already said, the Dirichlet control we are looking for is simply obtained by taking the trace of $u$ along $\Sigma_{T}$. As the trace on $\Sigma_{T}$ of $u \in H^{1}\left(Q_{T}\right)$, the control obtained then belongs to $H^{1 / 2}\left(\Sigma_{T}\right) \subset L^{2}\left(\Sigma_{T}\right)$. Neumann controls may be obtained in a similar way. Note that this argument implies that critical points can only occur at zero error.

Remark 2.1 We insist on the fact that this perspective relies on the minimization of the error functional, and does not make use of duality argument nor introduce any dual variable. For each $u$, the corrector $v$ is the solution of an elliptic linear and well-posed problem in $H^{1}\left(Q_{T}\right)$. Actually, this variational approach introduced by the second author in [30] is a least squares-type method, as deeply discussed, for instance, in [15, chap. VII], where the search of solution(s) for $F(u)=0$, given any $F: H_{0}^{1}(\Omega) \rightarrow H^{-1}(\Omega)$ and a bounded domain $\Omega \in \mathbb{R}^{N}$ is replaced by the extremal problem:

$$
\min _{u \in H_{0}^{1}(\Omega)}\|v\|_{H_{0}^{1}(\Omega)}^{2}
$$

where $v=v(u)$ solves the elliptic problem: $-\Delta v=F(u)$ in $\Omega, v=0$ on $\partial \Omega$.

We refer to [11] where a different variational approach leading to an elliptic problem defined on $Q_{T}$ has been introduced and analysed. 
Note that, even though there might not be rigorous results to be applied for some particular situation, the decrease of the error to zero is a sure indication that the problem is being (at least approximatively) controlled.

Remark 2.2 There are many ways to define the corrector $v$. One may, for instance, replace the state equation of (2.2) by the following equation:

$$
u_{t}-v_{t t}-\left(a(x) u_{x}+v_{x}\right)_{x}=0, \quad(x, t) \in Q_{T}
$$

leading to $E(u)=\frac{1}{2} \iint_{Q_{T}}\left(\left|v_{t}\right|^{2}+\left|v_{x}\right|^{2}\right) d x d t$, and the same expression of the first derivative. The choice we made in (2.2) seems the closest to the notion of a corrector for the heat equation.

\subsection{Inner controllability}

Let us now turn to the inner controllability case, which is as usual in control theory a bit simpler. This time we assume that the control is acting on a small subset $\omega$ (for simplicity, assumed independent of the time variable) of $(0,1)$.

Following again the ideas developed in the previous section, let us present an approach leading to a control in $L^{2}\left(q_{T}\right)$. We define the non-empty set

$$
\begin{aligned}
\mathscr{A}= & \left\{(u, f) ; u \in C\left([0, T] ; L^{2}(\Omega)\right) \cap L^{2}\left(0, T ; H_{0}^{1}(\Omega)\right) ; u^{\prime} \in L^{2}\left(0, T, H^{-1}(0,1)\right),\right. \\
& \left.\times u(x, 0)=u_{0}(x), u(x, T)=0, x \in(0,1), f \in L^{2}\left(q_{T}\right)\right\}
\end{aligned}
$$

and the extremal problem :

$$
\inf _{(u, f) \in \mathscr{A}} E(u, f):=\frac{1}{2} \iint_{Q_{T}}\left(\left|v_{t}\right|^{2}+a(x)\left|v_{x}\right|^{2}\right) d x d t
$$

where the corrector $v=v(u, f) \in H^{1}\left(Q_{T}\right)$ is defined by

$$
\left\{\begin{array}{lc}
u_{t}-v_{t t}-\left(a(x)\left(u_{x}+v_{x}\right)\right)_{x}-f 1_{\omega}=0, & (x, t) \in Q_{T}, \\
v_{t}(x, 0)=v_{t}(x, T)=0, & x \in(0,1), \\
v(0, t)=v(1, t)=0, & t \in(0, T) .
\end{array}\right.
$$

The well-posedness of this extremal problem is a consequence of the inner controllability of the heat equation: If $u$ is a controlled solution for the heat equation with a control $f \in L^{2}\left(q_{T}\right)$, then $(u, f)$ belongs to $\mathscr{A}, v$ solution of (2.2) vanishes on $Q_{T}$ and $\min _{(u, f) \in \mathscr{A}} E(u, f)=0$. 
Conversely, we check that any minimizer of $E$ in $\mathscr{A}$ is a solution of the heat equation with source term $f 1_{\omega}$. We define

$$
\begin{aligned}
\mathscr{A}_{0}= & \left\{(u, f) ; u \in C\left([0, T] ; L^{2}(\Omega)\right) \cap L^{2}\left(0, T ; H_{0}^{1}(\Omega)\right) ; u^{\prime} \in L^{2}\left(0, T, H^{-1}(0,1)\right),\right. \\
& \left.\times u(x, 0)=u(x, T)=0, x \in(0,1), f \in L^{2}\left(q_{T}\right)\right\}
\end{aligned}
$$

so that for any $(U, F) \in \mathscr{A}_{0}$, we compute that

$$
<E^{\prime}(u, f),(U, F)>=\iint_{Q_{T}}\left(v_{t} V_{t}+a(x) v_{x} V_{x}\right) d x d t=\iint_{Q_{T}}\left(U v_{t}-a(x) U_{x} v_{x}+F v 1_{\omega}\right) d x d t
$$

where $V \in H^{1}\left(Q_{T}\right)$ is the corrector associated with $(U, F) \in \mathscr{A}_{0}$. We have used here that $-\int_{0}^{T}<U_{t}, v>_{H^{-1}, H^{1}} d t=\iint_{Q_{T}} U v_{t} d x d t-\int_{0}^{1}[U v]_{0}^{T} d x=\iint_{Q_{T}} U v_{t} d x d t$. Writing that $<E^{\prime}(u, f),(U, F)>=0$ for all $(U, F)$ in $\mathscr{A}_{0}$, we obtain that the corrector satisfies the condition

$$
\begin{cases}v_{t}+\left(a(x) v_{x}\right)_{x}=0, & (x, t) \in Q_{T}, \\ v=0, & (x, t) \in q_{T}\end{cases}
$$

in addition to the boundary conditions on $v$ (see (2.8)). Again, a unique continuation property (see, for instance, [14, chap. 1]) implies that $v=0$ in $Q_{T}$ so that $(u, f) \in \mathscr{A}$ solves the heat equation. This reduces the search of a control $f$ distributed in $\omega$ to the minimization of the functional $E$ over $\mathscr{A}$.

Remark 2.3 In order to address the inner case, an alternative is as follows. Put $\mathscr{A}$ and $\mathscr{A}_{0}$ as in the boundary-controllability situation. To ensure that the solution $u$ satisfies the homogeneous heat equation off $q_{T}=\omega \times(0, T)$, we consider the following error functional:

$$
E(u)=\frac{1}{2} \iint_{Q_{T} \backslash q_{T}}\left(\left|v_{t}\right|^{2}+a(x)\left|v_{x}\right|^{2}\right) d x d t
$$

where the corrector $v$ is defined in two pieces:

(1) off $q_{T}$ :

$$
\left\{\begin{array}{lr}
u_{t}-v_{t t}-\left(a(x)\left(u_{x}+v_{x}\right)\right)_{x}=0, & (x, t) \in Q_{T} \backslash q_{T}, \\
v=0, & (x, t) \in \partial((0,1) \backslash \omega) \times(0, T), \\
v_{t}(x, 0)=v_{t}(x, T)=0, & x \in(0,1) \backslash \bar{\omega} .
\end{array}\right.
$$

(2) in $q_{T}$ :

$$
\left\{\begin{array}{lr}
u_{t}-v_{t t}-\left(a(x)\left(u_{x}+v_{x}\right)\right)_{x}=0, & (x, t) \in q_{T}, \\
v=0, & (x, t) \in \partial \omega \times(0, T), \\
v_{t}(x, 0)=v_{t}(x, T)=0, & x \in(0,1) \cap \bar{\omega} .
\end{array}\right.
$$

Then, proceeding as before, it can be shown that if $u$ is a minimizer for $E$, then the corresponding corrector $v$ vanishes outside $q_{T}$. A control is then given by $\left(v_{t t}+\left(a(x) v_{x}\right)_{x}\right) 1_{\omega}$. 
This procedure gives satisfactory numerical results. Note, however, that the control is only in $H^{-1}\left(q_{T}\right)$. However, this way of proceeding is a bit artificial in the sense that the control $u$ is found essentially as a boundary-controllability situation in $Q_{T} \backslash q_{T}$.

Remark 2.4 Since the controllability problem is formulated in $Q_{T}$, we may consider, without further change, the case where the support of the control depends on time variables, i.e. $q_{T}=\left\{(x, t) \in Q_{T}: g(t)<x<h(t), t \in(0, T)\right\}$, where $g$ and $h$ are two smooth functions on $[0, T]$ with $0<g \leqslant h<1, g(t) \neq h(t)$. We refer to [11] for some experiments using a different variational approach.

\section{Convergence of minimizing sequences for $E$}

One main issue in all the situations discussed earlier is to establish the convergence of the various numerical procedures proposed. They all seem to perform reasonably well, at least in the numerical tests carried out in this contribution. One main difficulty in showing such convergence of iterates is that the error functionals are not, in general, coercive in the appropriate spaces. They all are quadratic, non-negative, and so convex. But we will, in general, lack coercivity and strict convexity. To prove the convergence of all the numerical procedures treated here, we prove a general-purpose result that can be applied to the various scenarios dealt with above.

Lemma 3.1 Suppose $\mathbf{T}: X \mapsto Y$ is a linear, continuous operator between Hilbert spaces, and $H \subset X$, a closed subspace, $u_{0} \in X$. Put

$$
E: u_{0}+H \mapsto \mathbf{R}^{+}, E(u)=\frac{1}{2}\|\mathbf{T} u\|^{2}, \quad A=k e r \mathbf{T} \cap H .
$$

(1) Then $E: u_{0}+A^{\perp} \rightarrow \mathbf{R}$ is quadratic, non-negative and strictly convex, where $A^{\perp}$ is the orthogonal complement of $A$ in $H$.

(2) The derivative $E^{\prime}\left(u_{0}+u\right)$ always belongs to $A^{\perp}$. In particular, a typical steepest descent procedure will always stay in the manifold $u_{0}+A^{\perp}$.

(3) If, in addition,

$$
\min _{u \in H} E\left(u_{0}+u\right)=0,
$$

then the steepest descent scheme will always produce sequences converging (strongly in $X$ ) to a unique (in $u_{0}+A^{\perp}$ ) minimizer $u_{0}+\bar{u}$ with zero error.

Proof Suppose there are $u_{i} \in A^{\perp}, i=1,2$, such that

$$
E\left(u_{0}+\frac{1}{2} u_{1}+\frac{1}{2} u_{2}\right)=\frac{1}{2} E\left(u_{0}+u_{1}\right)+\frac{1}{2} E\left(u_{0}+u_{2}\right) .
$$

Due to the strict convexity of the norm in a Hilbert space, we deduce that this equality can only occur if $\mathbf{T} u_{1}=\mathbf{T} u_{2}$. Therefore, $u_{1}-u_{2} \in A \cap A^{\perp}=\{0\}$, and $u_{1}=u_{2}$. 
For the second part, note that for arbitrary $U \in A, \mathbf{T} U=0$, and so

$$
E\left(u_{0}+u+U\right)=\frac{1}{2}\left\|\mathbf{T} u_{0}+\mathbf{T} u+\mathbf{T} U\right\|^{2}=\frac{1}{2}\left\|\mathbf{T} u_{0}+\mathbf{T} u\right\|^{2}=E\left(u_{0}+u\right) .
$$

Therefore, the derivative $E^{\prime}\left(u_{0}+u\right)$, the steepest descent direction for $E$ at $u_{0}+u$, has to be orthogonal to all such $U \in A$.

Finally, assume $E\left(u_{0}+\bar{u}\right)=0$. It is clear that this minimizer is unique in $u_{0}+A^{\perp}$ (recall the strict convexity in (1)). This, in particular, implies that for arbitrary $u \in A^{\perp}$,

$$
\left\langle E^{\prime}\left(u_{0}+u\right), \bar{u}-u\right\rangle \leqslant 0
$$

because this inner product is the derivative of the section $t \mapsto E\left(u_{0}+t \bar{u}+(1-t) u\right)$ at $t=0$, and this section must be a positive parabola with the minimum point at $t=1$. If we consider the gradient flow

$$
u^{\prime}(t)=-E^{\prime}\left(u_{0}+u(t)\right), \quad t \in[0,+\infty)
$$

then, because of (3.1),

$$
\frac{d}{d t}\left(\frac{1}{2}\|u(t)-\bar{u}\|^{2}\right)=\left\langle u(t)-\bar{u}, u^{\prime}(t)\right\rangle=\left\langle u(t)-\bar{u},-E^{\prime}\left(u_{0}+u(t)\right)\right\rangle \leqslant 0 .
$$

This implies that sequences produced through the steepest descent method will be minimizing for $E$, uniformly bounded in $X$ (because $\|u(t)-\bar{u}\|$ is a non-increasing function of $t$ ), and due to the strict convexity of $E$ restricted to $u_{0}+A^{\perp}$, they will have to converge towards the unique minimizer $u_{0}+\bar{u}$.

Remark 3.2 Despite the strong convergence in this statement, it may not be true that the error is coercive, even restricted to $u_{0}+A^{\perp}$, so that that strong convergence could be very slow. Because of this same reason, it may be impossible to establish rates of convergence for these minimizing sequences.

The element $u^{0}$ determines the non-homogeneous data set of each problem: source term, boundary conditions, initial and/or final condition etc. The subspace $H$ is the subset of the ambient Hilbert space $X$ for which the data set vanishes. $T$ is the operator defining the corrector so that $\operatorname{ker}(T)$ is the subspace of all solutions of the underlying equation or system. The subspace $A$ is the subspace of all solutions of the problem with vanishing data set. In some situations $A$ will be trivial, but in some others will not be so. The important property is (3) in the statement guaranteeing that we indeed have strong convergence in $X$ of iterates. The main requirement for this is to know a priori that the error attains its minimum value, i.e. zero somewhere.

In the particular situation of boundary controllability for the heat equation (Section 2.1), $X$ is taken to be $H^{1}\left(Q_{T}\right)$, and $H=\mathscr{A}_{0}$ is given in (2.4). The operator $\mathbf{T}$ takes each $u \in H^{1}\left(Q_{T}\right)$ into its corrector $v$ through (2.2). If $u^{0}$ is chosen appropriately, then $u^{0}+H=\mathscr{A}$ in (2.1). Finally, the subspace $A$ is the subspace of all solutions of the homogeneous heat equation which also comply with the vanishing boundary conditions 
around the boundary of $Q_{T}$ except for $x=1$. This is a non-trivial subspace of $H^{1}\left(Q_{T}\right)$ that makes an interesting problem to look for the control of minimal $L^{2}$-norm over $x=1$. As has already pointed out, we do know that the error does attain the minimum value, and it vanishes because exact boundary null-controllability holds for the heat equation. Therefore, the main requirement of the above lemma holds, and we thus have the strong convergence of computed iterative sequences based on the minimization of the error.

\section{Numerical resolution of the minimization problem}

As shown in the previous section, the practical search of controls for the heat equation may be reduced to the minimization for corrector problems. We describe in this section the minimization procedure to approximate numerically correctors. We give the details of the boundary case, and then point out the main differences for the inner counterpart.

For the boundary case, we have to solve

$$
\begin{cases}\text { Minimize } & E(u)=\frac{1}{2} \iint_{Q_{T}}\left(\left|v_{t}\right|^{2}+a(x)\left|v_{x}\right|^{2}\right) d x d t \\ \text { subject to } \quad u \in \mathscr{A}\end{cases}
$$

with $\mathscr{A}=\left\{u \in H^{1}\left(Q_{T}\right) ; u(x, 0)=u_{0}\right.$ on $(0,1), u(\cdot, T)=0$ on $(0,1), u(x, t)=0$ on $\left.\Sigma_{T}\right\}$. We endowed the space $\mathscr{A}$ with the scalar product

$$
(u, v)_{\mathscr{A}}=\iint_{Q_{T}}\left(u_{t} v_{t}+a(x) u_{x} v_{x}+u v\right) d x d t, \quad \forall u, v \in \mathscr{A}
$$

and note that $\|u\|_{\mathscr{A}}=\sqrt{(u, u)_{\mathscr{A}}}$ for all $u \in \mathscr{A}$. The Hilbert space $\mathscr{A}_{0}$ is endowed with the same scalar product.

\subsection{Conjugate gradient algorithm}

The Polak-Ribière version of the $\mathrm{CG}$ algorithm to minimize $E$ over $\mathscr{A}$ is as follows (see [15]):

- Step 0. Initialization: Given any $\varepsilon>0$ and any $u^{0} \in \mathscr{A}$, compute the residual $g^{0} \in \mathscr{A}_{0}$ solution of

$$
\left(g^{0}, U\right)_{\mathscr{A}}=<E^{\prime}\left(u^{0}\right), U>\quad \forall U \in \mathscr{A}_{0} .
$$

If $\left\|g^{0}\right\| /\left\|u^{0}\right\| \leqslant \varepsilon$, take $u=u^{0}$ as an approximation of a minimum of $E$, otherwise set $z^{0}=g^{0}$.

For $n \geqslant 0$, assuming $u^{n}, g^{n}, z^{n}$ being known with $g^{n}$ and $z^{n}$, both different from zero, compute $u^{n+1}, g^{n+1}$ and if necessary $z^{n+1}$ as follows:

- Step 1. Steepest descent: Set $u^{n+1}=u^{n}-\lambda_{n} z^{n}$, where $\lambda_{n} \in \mathbb{R}$ is the solution of the $1 \mathrm{D}$ minimization problem,

$$
\text { minimize } E\left(u^{n}-\lambda z^{n}\right), \quad \text { over } \lambda \in \mathbb{R} \text {. }
$$


Then compute the residual $g^{n+1} \in \mathscr{A}_{0}$ from the relation

$$
\left(g^{n+1}, U\right)_{\mathscr{A}}=<E^{\prime}\left(u^{n+1}\right), U>\quad \forall U \in \mathscr{A}_{0} .
$$

- Step 2. Convergence testing and construction of the new descent direction: If $\left\|g^{n+1}\right\|_{\mathscr{A}} /\left\|g^{0}\right\|_{\mathscr{A}} \leqslant \varepsilon$, take $u=u^{n+1}$; otherwise compute

$$
\gamma_{n}=\frac{\left(g^{n+1}, g^{n+1}-g^{n}\right)_{\mathscr{A}}}{\left(g^{n}, g^{n}\right)_{\mathscr{A}}}, \quad z^{n+1}=g^{n+1}+\gamma_{n} z^{n} .
$$

Then do $n=n+1$, and return to step 1 .

Let us provide more details for two important steps of the algorithm:

- Since $E$ is a quadratic functional with respect to $u$, one may explicitly solve problem (4.2): We write

$$
\begin{aligned}
E\left(u^{n}-\lambda z^{n}\right)= & E\left(u^{n}\right)-\lambda \iint_{Q_{T}}\left(v_{t}^{n} Z_{t}^{n}+a(x) v_{x}^{n} Z_{x}^{n}\right) d x d t \\
& +\frac{\lambda^{2}}{2} \iint_{Q_{T}}\left(\left|Z_{t}^{n}\right|^{2}+a(x)\left|Z_{x}^{n}\right|^{2}\right) d x d t
\end{aligned}
$$

where $Z_{x}^{n}$ is the corrector of $z^{n}$, solution of

$$
\left\{\begin{array}{l}
z_{t}^{n}-Z_{t t}^{n}-\left(a(x)\left(z_{x}^{n}+Z_{x}^{n}\right)\right)_{x}=0, \quad(x, t) \in Q_{T}, \\
Z(\cdot, t)=0, \quad \Sigma_{t}, \quad Z_{t}(x, \cdot)=0, \quad \Sigma_{x}
\end{array}\right.
$$

so that the optimal parameter is given by

$$
\lambda_{n}=\frac{\iint_{Q_{T}}\left(v_{t}^{n} Z_{t}^{n}+a(x) v_{x}^{n} Z_{x}^{n}\right) d x d t}{\iint_{Q_{T}}\left(\left|Z_{t}^{n}\right|^{2}+a(x)\left|Z_{x}^{n}\right|^{2}\right) d x d t}=-\frac{\iint_{Q_{T}}\left(z_{t}^{n} v^{n}+a(x) z_{x}^{n} v_{x}^{n}\right) d x d t}{\iint_{Q_{T}}\left(\left|Z_{t}^{n}\right|^{2}+a(x)\left|Z_{x}^{n}\right|^{2}\right) d x d t}
$$

- The computation of the residual $g^{n}$ is performed as follows. According to equality

$$
<E^{\prime}\left(u^{n}\right), U>=-\iint_{Q_{T}}\left(U_{t} v^{n}+a(x) U_{x} v_{x}^{n}\right) d x d t, \quad \forall U \in \mathscr{A}_{0},
$$

$\left.E^{\prime}\left(u^{n}\right) \in H^{-1}\left(Q_{T}\right)\right)$ may be identified with the linear functional on $\mathscr{A}_{0}$ defined by

$$
U \rightarrow-\iint_{Q_{T}}\left(U_{t} v^{n}+a(x) U_{x} v_{x}^{n}\right) d x d t
$$

It then follows that $g^{n}$ is the solution of the following linear variational problem: Find $g^{n} \in \mathscr{A}_{0}$ such that

$$
\iint_{Q_{T}}\left(g_{t}^{n} U_{t}+a(x) g_{x}^{n} U_{x}+g^{n} U\right) d x d t=-\iint_{Q_{T}}\left(U_{t} v^{n}+a(x) U_{x} v_{x}^{n}\right) d x d t, \quad \forall U \in \mathscr{A}_{0},
$$


where $v^{n} \in H_{0, x}^{1}\left(Q_{T}\right)$ is the corrector associated with $u^{n}$. The well-posed elliptic boundary value problem corresponding to this variational formulation is

$$
\left\{\begin{array}{lc}
-g_{t t}^{n}-\left(a(x) g_{x}^{n}\right)_{x}+g^{n}=v_{t}^{n}+\left(a(x) v_{x}^{n}\right)_{x} & (x, t) \in Q_{T}, \\
g^{n}(0, t)=0, g_{x}^{n}(1, t)+v_{x}^{n}(1, t)=0, & t \in(0, T), \\
g^{n}(x, 0)=g^{n}(x, T)=0, & x \in(0,1) .
\end{array}\right.
$$

Remark 4.1 As we have mentioned above, the parameter $\gamma_{n}$ given by (4.3) corresponds to the Polak-Ribière version of the conjugate gradient algorithm. In the present quadraticlinear situation, this one should coincide with the Fletcher-Reeves conjugate algorithm for which

$$
\gamma_{n}=\left\|g^{n+1}\right\|_{\mathscr{A}}^{2} /\left\|g^{n}\right\|_{\mathscr{A}}^{2},
$$

since gradients are conjugate to each other $\left(\left(g^{m}, g^{n}\right)_{\mathscr{A}}=0\right.$ for all $\left.m \neq n\right)$. However, we observed that in the parabolic situation (see also [11]) the Polak-Ribière version (mainly used in nonlinear situations) allows to reduce the loss of orthogonality due to numerical approximation.

The detailed conjugate gradient scheme, written in a variational form, used for the minimization of $E$ is then as follows:

Step 0. Initialization: $u^{0} \in \mathscr{A}$ be given, compute the corrector $v^{0} \in H_{0, x}^{1}\left(Q_{T}\right)$ of $u^{0}$ solution of

$$
\iint_{Q_{T}}\left(v_{t}^{0} \phi_{t}+a(x) v_{x}^{0} \phi_{x}\right) d x d t=-\iint_{Q_{T}}\left(u_{t}^{0} \phi+a(x) u_{x}^{0} \phi_{x}\right) d x d t, \quad \forall \phi \in H_{0, x}^{1}\left(Q_{T}\right),
$$

then compute the gradient $g^{0} \in \mathscr{A}_{0}$ solution of

$$
\iint_{Q_{T}}\left(g_{t}^{0} \phi_{t}+a(x) g_{x}^{0} \phi_{x}+g^{0} \phi\right) d x d t=-\iint_{Q_{T}}\left(v^{0} \phi_{t}+a(x) v_{x}^{0} \phi_{x}\right) d x d t, \quad \forall \phi \in \mathscr{A}_{0},
$$

and set $z^{0}=g^{0}$.

Then, for $n \geqslant 0$, assuming $u^{n}, g^{n}, z^{n}$ and $v^{n}$ known, compute $u^{n+1}, g^{n+1}, z^{n+1}$ and $v^{n+1}$ by:

Step 1. Steepest descent: Compute the corrector $Z^{n} \in H_{0, x}^{1}\left(Q_{T}\right)$ of $z^{n}$ solution

$$
\iint_{Q_{T}}\left(Z_{t}^{n} \phi_{t}+a(x) Z_{x}^{n} \phi_{x}\right) d x d t=-\iint_{Q_{T}}\left(z_{t}^{n} \phi+a(x) z_{x}^{n} \phi_{x}\right) d x d t, \quad \forall \phi \in H_{0, x}^{1}\left(Q_{T}\right),
$$

and set $u^{n+1}=u^{n}-\lambda_{n} z^{n} \in \mathscr{A}$ with

$$
\lambda_{n}=-\frac{\iint_{Q_{T}}\left(z_{t}^{n} v^{n}+a(x) z_{x}^{n} v_{x}^{n}\right) d x d t}{\iint_{Q_{T}}\left(\left|Z_{t}^{n}\right|^{2}+a(x)\left|Z_{x}^{n}\right|^{2}\right) d x d t}
$$


Next, compute the corrector $v^{n+1} \in \mathscr{A}_{1}$ of $u^{n+1}$ solution of

$$
\iint_{Q_{T}}\left(v_{t}^{n+1} \phi_{t}+a(x) v_{x}^{n+1} \phi_{x}\right) d x d t=-\iint_{Q_{T}}\left(u_{t}^{n+1} \phi+a(x) u_{x}^{n+1} \phi_{x}\right) d x d t, \quad \forall \phi \in H_{0, x}^{1}\left(Q_{T}\right),
$$

and the gradient $g^{n+1} \in \mathscr{A}_{0}$ solution of

$$
\iint_{Q_{T}}\left(g_{t}^{n+1} \phi_{t}+a(x) g_{x}^{n+1} \phi_{x}+g^{n+1} \phi\right) d x d t=-\iint_{Q_{T}}\left(v^{n+1} \phi_{t}+a(x) v_{x}^{n+1} \phi_{x}\right) d x d t, \quad \forall \phi \in \mathscr{A}_{0} .
$$

Step 2. Construction of the new descent direction: If $\left\|g^{n+1}\right\|_{\mathscr{A}} /\left\|g^{0}\right\|_{\mathscr{A}} \leqslant \varepsilon$, take $u=u^{n+1}$; otherwise compute

$$
\gamma_{n}=\frac{\left(g^{n+1}, g^{n+1}-g^{n}\right)_{\mathscr{A}}}{\left(g^{n}, g^{n}\right)_{\mathscr{A}}}, \quad z^{n+1}=g^{n+1}+\gamma_{n} z^{n} .
$$

Then do $n=n+1$, and return to step 1 .

Once the convergence of the algorithm is reached, up to the threshold $\varepsilon$, we take the trace of $u$ on $\Sigma_{T}$ to define an approximation of the control $w$ of $(1.3): w(t)=u(1, t)$, $t \in(0, T)$. We next compute an approximation of the controlled solution $u$ by solving (1.3): the $L^{2}$-norm $\|u(\cdot, T)\|_{L^{2}(0,1)}$, which may be seen as an a posteriori error, allows to evaluate the efficiency of the approach.

The minimization of the functional $E$ related to the inner case (see (2.9)) is very similar. The main difference is that the corrector have to be solved independently in and off $q_{T}$ (see (2.10) and (2)). The additional condition is $v=0$ on $\partial \omega \times(0, T)$. It is important to note that these correctors are linked through the descent direction $g^{n}$, solution of the problem posed in all of the domain $Q_{T}$ :

$$
\begin{aligned}
\iint_{Q_{T}}\left(g_{t}^{n} U_{t}+a(x) g_{x}^{n} U_{x}+g^{n} U\right) d x d t & =-\iint_{Q_{T} \backslash q_{T}}\left(U_{t} v^{n}+a(x) U_{x} v_{x}^{n}\right) d x d t, \quad \forall U \in \mathscr{A}_{0} \\
& =H_{0}^{1}\left(Q_{T}\right) .
\end{aligned}
$$

\subsection{Numerical approximation}

For 'large' integers $N_{x}$ and $N_{t}$, we set $\Delta x=1 / N_{x}, \Delta t=T / N_{t}$ and $h=(\Delta x, \Delta t)$. Let us denote by $\mathscr{P}_{\Delta x}$ the uniform partition of $[0,1]$ associated with $\Delta x$, and let us denote by $\mathscr{Q}_{h}$ the uniform quadrangulation of $Q_{T}$ associated with $h$. In particular,

$$
\bar{Q}_{T}=\bigcup_{K \in \mathscr{Q}_{h}} K
$$

The following (conformal) finite element approximation of $H^{1}\left(Q_{T}\right)$ is introduced:

$$
X_{h}=\left\{\varphi_{h} \in C^{0}([0,1] \times[0, T]):\left.\varphi_{h}\right|_{K} \in\left(\mathbb{P}_{1, x} \otimes \mathbb{P}_{1, t}\right)(K) \quad \forall K \in \mathscr{Q}_{h}\right\} .
$$

Here $\mathbb{P}_{m, \xi}$ denotes the space of polynomial functions of order $m$ in the variable $\xi$. Accordingly, the functions in $X_{h}$ reduce on each quadrangle $K \in \mathscr{Q}_{h}$ to a polynomial of 
the form $A+B x+C t+D x t$ involving four degrees of freedom (4 DOF)). Obviously, the space $X_{h}$ is a conformal approximation of $L^{2}\left(Q_{T}\right)$. We will also consider the space

$$
\begin{aligned}
& X_{0 h}=\left\{\varphi_{h} \in X_{h}: \varphi_{h}(0, t)=\varphi_{h}(1, t)=0 \quad \forall t \in(0, T)\right\}, \\
& X_{u h}=\left\{\varphi_{h} \in X_{h}: \varphi_{h}(0, t)=0 \quad \forall t \in(0, T), \varphi_{h}(x, 0)=u_{0}(x), \varphi_{h}(x, T)=0 \quad \forall x \in(0,1)\right\} .
\end{aligned}
$$

$X_{u h}$ and $X_{0 h}$ are finite-dimensional subspace of $\mathscr{A}$ and $H_{0, x}^{1}\left(Q_{T}\right)$ respectively (and also of $\left.L^{2}\left(0, T ; H^{1}(0,1)\right)\right)$. Functions $\varphi_{h} \in X_{0 h}$ are uniquely determined by their values at the nodes $\left(x_{j}, t_{j}\right)$ of $\mathscr{Q}_{h}$ such that $0<x_{j}<1$.

Therefore, for any $h$, we consider the following problem, which is an approximation of (4.1):

$$
\begin{cases}\text { Minimize } & E_{h}\left(u_{h}\right)=\frac{1}{2} \iint_{Q_{T}}\left(\left|v_{h, t}\right|^{2}+a(x)\left|v_{h, x}\right|^{2}\right) d x d t, \\ \text { subject to } \quad u_{h} \in X_{u h} .\end{cases}
$$

According to the conjugate gradient algorithm, this minimization problem is reduced to the resolution of well-posed elliptic problems defined on $Q_{T}$ in order to compute corrector functions $v_{h} \in X_{0 h}$.

Once the optimal function $u_{h}$, minimizer of $E$ over $X_{h}$, is obtained, the control $w_{h}$ is defined by $w_{h}=u_{h}$ on $\Sigma_{T}$. In order to check the quality of the control $w_{h}$, piecewise linear along $\Sigma_{T}$, one may compare such solution in $X_{u h}$ with the solution $\bar{u}_{h}$ of (1.3) starting from $u_{0}$ at time $t=0$ such that $\bar{u}_{h}=w_{h}$ on $\Sigma_{T} . \bar{u}_{h}$ is computed using, for the time discretization, the two-step implicit Gear scheme of order two in time (see, for instance, [16]). We set

$$
\Phi_{\Delta x}=\left\{z \in C^{0}([0,1]):\left.z\right|_{k} \in \mathbb{P}_{1, x}(k) \quad \forall k \in \mathscr{P}_{\Delta x}\right\}
$$

a finite-dimensional subspace of $L^{2}(0,1)$. Functions in $\Phi_{\Delta x}$ are uniquely determined by their values at the nodes of $\mathscr{P}_{\Delta x}$.

The Gear scheme, which is of order two, is then combined with a $\mathbb{P}_{1}$-finite element discretization in space as follows:

(1) Firstly, we first set $\left.u_{h}\right|_{t=0}=u_{0, \Delta x}$.

(2) Secondly, $\left.u_{h}\right|_{t=t_{1}}$ is the solution of the linear problem in $\Psi \in \Phi_{\Delta x}$

$$
\left\{\begin{array}{l}
\int_{0}^{1} \frac{1}{\Delta t}\left(\Psi-\left.\bar{u}_{h}\right|_{t=0}\right) z d x+\frac{1}{2} \int_{0}^{1} a(x)\left(\Psi+\left.\bar{u}_{h}\right|_{t=0}\right)_{x} z_{x} d x=0 \\
\forall z \in \Phi_{\Delta x} .
\end{array}\right.
$$

(3) For given $n=2, \ldots, N_{t}-1, \Psi^{\star}=\left.\bar{u}_{h}\right|_{t=t_{n-1}}$ and $\bar{\Psi}=\left.\bar{u}_{h}\right|_{t=t_{n}},\left.\bar{u}_{h}\right|_{t=t_{n+1}}$ is the solution of the linear problem in $\Psi \in \Phi_{\Delta x}$

$$
\left\{\begin{array}{l}
\int_{0}^{1} \frac{1}{2 \Delta t}\left(3 \Psi-4 \bar{\Psi}+\Psi^{\star}\right) z d x+\int_{0}^{1} a(x) \Psi_{x} z_{x} d x=0 \\
\forall z \in \Phi_{\Delta x}
\end{array}\right.
$$

The $L^{2}$-norm $\left\|u_{h}(\cdot, T)-\bar{u}_{h}(\cdot, T)\right\|_{L^{2}(0,1)}=\left\|\bar{u}_{h}(\cdot, T)\right\|_{L^{2}(0,1)}$ allows to analyse a posteriori how 
Table 1. Boundary case $-u_{0}(x)=\sin (\pi x), T=1 / 2, a_{0}=1 / 4, \Delta x=\Delta t=1 / 100-$ $\varepsilon=10^{-5}-$ numerical results with respect to $h=(\Delta x, \Delta t)$

\begin{tabular}{lcccc}
\hline \hline$\Delta x=\Delta t$ & $1 / 25$ & $1 / 50$ & $1 / 100$ & $1 / 200$ \\
\hline \# CG iteration & 846 & 2,132 & 2,014 & 2,834 \\
$\left\|u_{h}\right\|_{L^{2}\left(Q_{T}\right)}$ & $4.78 \times 10^{-1}$ & $5.06 \times 10^{-1}$ & $4.81 \times 10^{-1}$ & $4.87 \times 10^{-1}$ \\
$\left\|u_{h}\right\|_{H^{1}\left(Q_{T}\right)}$ & 6.024 & 6.658 & 5.920 & 6.021 \\
$\left\|u_{h}\right\|_{L^{2}\left(\Sigma_{T}\right)}$ & 1.369 & 1.487 & 1.392 & 1.418 \\
$\left\|\bar{u}_{h}(\cdot, T)\right\|_{L^{2}(0,1)}$ & $1.95 \times 10^{-2}$ & $9.65 \times 10^{-3}$ & $8.39 \times 10^{-3}$ & $6.04 \times 10^{-3}$ \\
$\left\|u_{h}-\bar{u}_{h}\right\|_{L^{2}\left(Q_{T}\right)}$ & $1.45 \times 10^{-2}$ & $6.31 \times 10^{-3}$ & $2.01 \times 10^{-3}$ & $9.34 \times 10^{-4}$ \\
$E_{h}\left(u_{h}\right)$ & $4.88 \times 10^{-6}$ & $8.37 \times 10^{-7}$ & $1.22 \times 10^{-6}$ & $8.29 \times 10^{-7}$ \\
\hline \hline
\end{tabular}

the constraint (1.4) is satisfied. Recall that $\bar{u}_{h}$, obtained by an integration in time, solves the heat equation.

This same numerical approximation is used for the inner case.

\section{Numerical experiments}

We now present some numerical experiments, and analyse the behaviour of the computed controls with respect to the data, and $h$. We assume for simplicity that $\Delta x=\Delta t$, that is, we consider only uniform meshes $\mathscr{Q}_{h}$.

\subsection{Experiment 1: boundary case}

As in $[11,29]$, we assume that the function $u_{0}$ to be controlled is the first mode of the Laplacian, that is,

$$
u_{0}(x)=\sin (\pi x), \quad x \in(0,1)
$$

for which the diffusion of (1.3), without control, i.e. $v=0$, is the lowest. Moreover, we assume that the diffusion function $a$ is constant equal to $a(x)=a_{0}=1 / 4$ in $(0,1)$, and take a controllability time, $T=1 / 2$. We take a value $a_{0}$ lower than one in order to have a better control of the diffusion. Without control, these data lead to $\|u(\cdot, T)\|_{L^{2}(0,1)}=$ $\sqrt{1 / 2} e^{-\pi^{2} / 8} \approx 0.205$ and therefore leads to a stiff case in the context of null boundary controllability for the heat equation.

We take $\varepsilon=10^{-5}$ as the value for the stopping criterion of the conjugate gradient algorithm. The algorithm is initialized with $u^{0} \in \mathscr{A}$ defined by $u^{0}(x, t)=u_{0}(x)(1-t / T)^{2}$, $(x, t) \in Q_{T}$.

Table 1 gives various norms of the solution $u_{h} \in \mathscr{A}$ with respect to $h$, and clearly suggests the convergence of the approximation. Figure 1 depicts the evolution of $E_{h}\left(u_{h}^{n}\right)$ and the residual $\left\|g_{h}^{n}\right\|_{\mathscr{A}}$ (in $\log _{10}$-scale) with respect to the iteration of the conjugate gradient corresponding to $\Delta x=\Delta t=1 / 100$. The algorithm requires 2,013 iterations to fulfill $\left\|g_{h}^{n}\right\|_{\mathscr{A}} \leqslant \varepsilon$. As is typical when the heat equation is involved, the slope of the residual decreases significantly after the first iteration. This phenomenon is also possibly due to the lack of coercivity of $E$. We, however, check that the functional $E_{h}\left(u_{h}^{n}\right)$ decreases with respect to the iteration and reaches a small value, here of the order $\mathcal{O}\left(10^{-6}\right)$. 


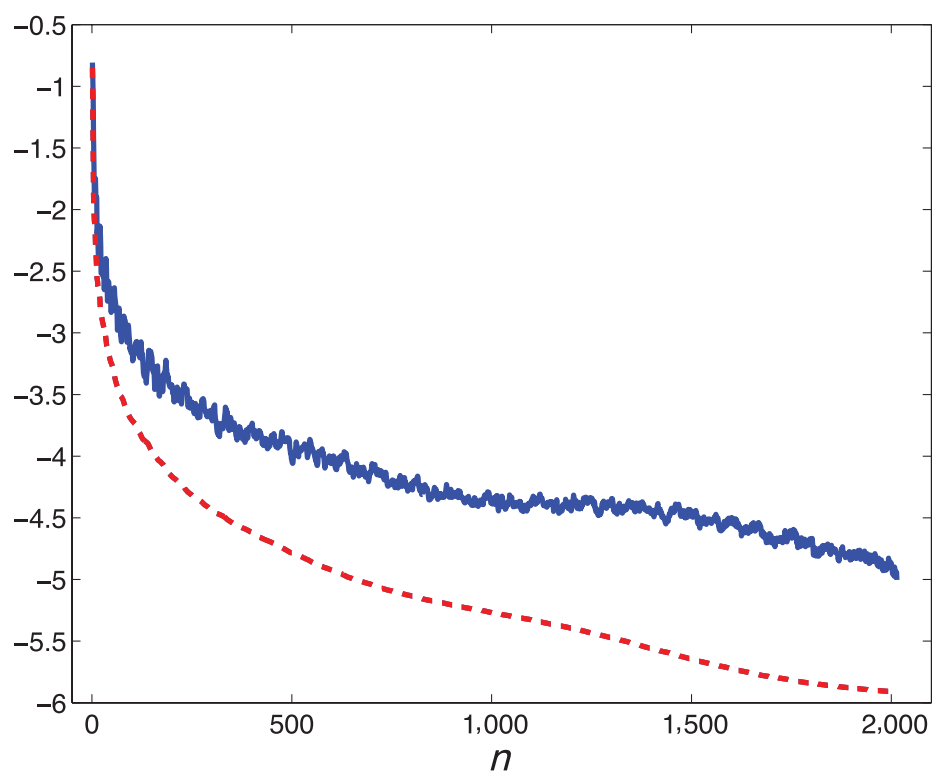

Figure 1. (Colour online) Boundary case $-u_{0}(x)=\sin (\pi x), \Delta x=\Delta t=1 / 100-\log _{10}\left(E_{h}\left(u_{h}^{n}\right)\right)$ (dashed line) and $\log _{10}\left(\left\|g_{h}^{n}\right\|_{\mathscr{A}}\right.$ ) (full line) versus the iteration $n$ of the conjugate gradient algorithm.

Figure 2 depicts the corresponding solution $u_{h} \in \mathscr{A}$, and corrector $v_{h} \in H_{0, x}^{1}\left(Q_{T}\right)$. The trace of $u_{h}$ on $\Sigma_{T}$ is given in Figure 4 (left). The control obtained is oscillating near $t=T$. This is reminiscent of what is obtained in [20] by computing exactly the controlled heat solution in the 1D space by means of the motion planning method (we also refer to [29] for an adaptation to the inner case using the so-called transmutation method). We also plot in Figure 3 the iso-values of the solution $u_{h}$ that allow to appreciate the diffusion and the control of the heat from the initial to the controllability time. Finally, the solution $\bar{u}_{h}$ of (1.3) with $w(t)=u_{h}(1, t)$ is plotted at time $T$ in Figure 4 (right). We compute that the $L^{2}$-norm of $\bar{u}_{h}(1, T)$, what we called the a posteriori error, is $\left\|\bar{u}_{h}(\cdot, T)\right\|_{L^{2}(0,1)} \approx 8.39 \times 10^{-3}$. This is an acceptable value that can be improved by reducing $\varepsilon$ and $h$. Note that the stiffness matrices involved in the resolution of the elliptic problems in step 1 are standard and well-conditioned. Note also that a small gap between $u$ and $\bar{u}$ (in particular at time $T$ ) is a priori unavoidable since they are approximated and computed in a different way.

It is also interesting to note that this method allows to obtain non-trivial controlled solution of the heat equation with zero initial data, that is in $\mathscr{A}_{0}$. Figure 5 depicts one such solution obtained with the initial function $u^{0}(x, t)=\sin (\pi x) t^{2}(1-t / T)^{2}$. For $\varepsilon=10^{-6}$, the algorithm converges after 1,242 iterations, and we get $E_{h}\left(u_{h}^{n=1242}\right) \approx 6.63 \times 10^{-9}$ and $\left\|\bar{u}_{h}(\cdot, T)\right\|_{L^{2}(0,1)} \approx 2.89 \times 10^{-5}$. Accordingly, this means that any linear combination of such non-trivial solution in $\mathscr{A}_{0}$ with the previous ones in $\mathscr{A}$ remains a controlled solution of the heat equation. We will get back to this notion in Section 7. The non-uniqueness of our minimization problem may also be checked by considering different initial function $u^{0} \in \mathscr{A}$.

As expected, the experiments also suggest that the situation is more favourable, notably with respect to the speed of convergence of the algorithm when the control acts on both 

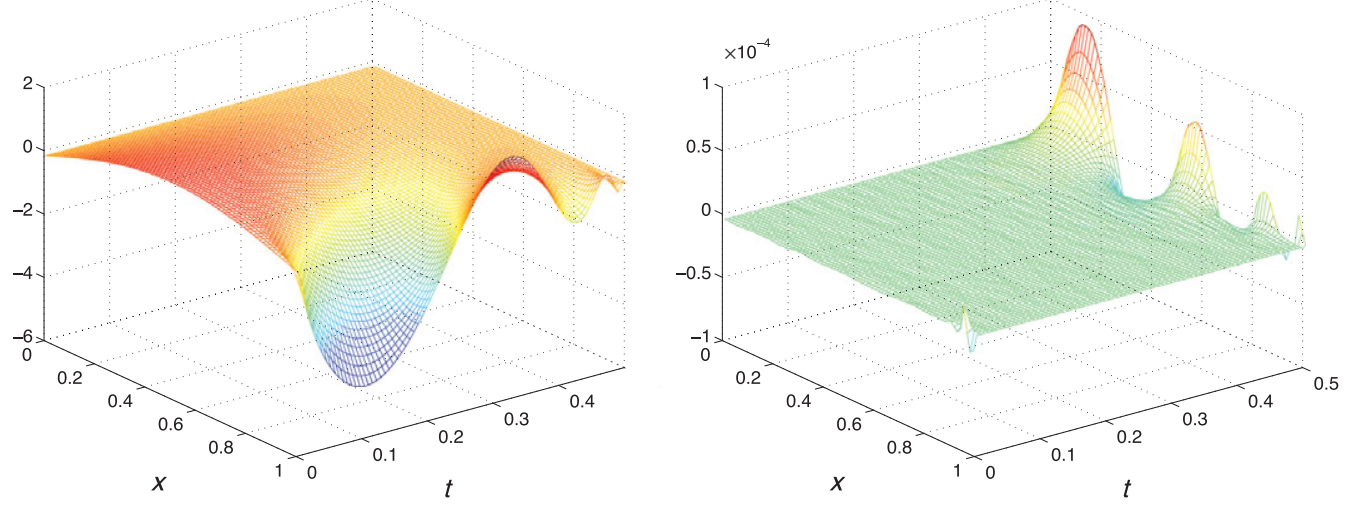

Figure 2. (Colour online) Boundary case $-u_{0}(x)=\sin (\pi x)$. Solution of $u_{h} \in \mathscr{A}_{h}$ (left) and corresponding corrector $v_{h}$ (right) along $Q_{T}$.

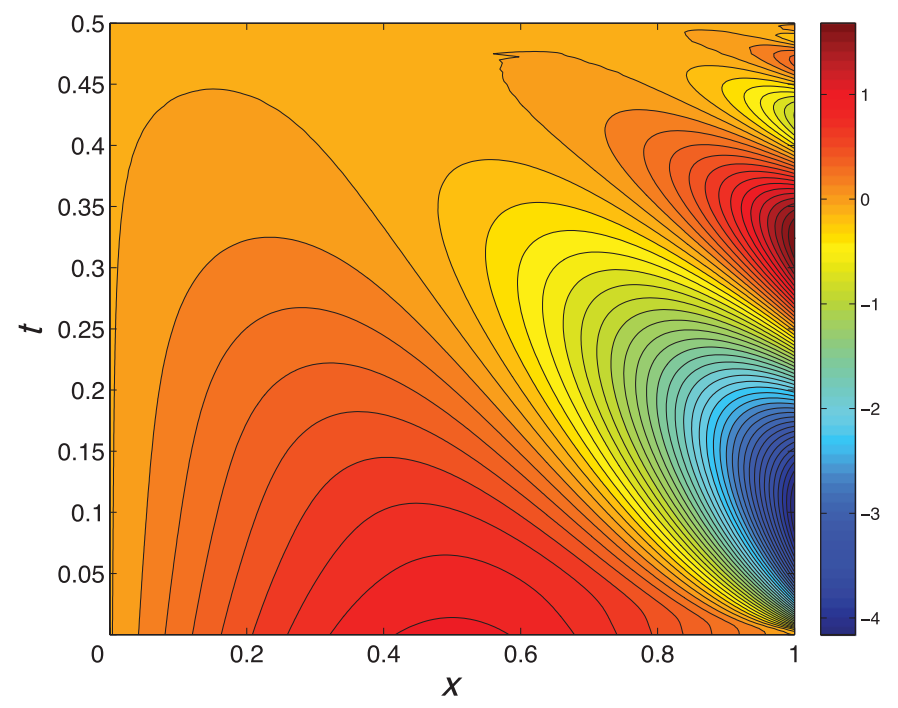

Figure 3. (Colour online) Boundary case $-u_{0}(x)=\sin (\pi x)$. Iso-values of $u_{h} \in \mathscr{A}_{h}$ along $Q_{T}$.

sides, that is, on $x=0$ and 1 . Figure 6 shows the controlled solution with initial data $u_{0}(x)=\sin (\pi x)+\sin (2 \pi x)+\sin (3 \pi x)$ in that situation. For the same value of $\varepsilon$, the $L^{2}$-norm of the corrector as well as the a posteriori error are lower than in the previous situation: $E_{h}\left(u_{h}^{n=855}\right) \approx 6.69 \times 10^{-6}$ and $\left\|\bar{u}_{h}(\cdot, T)\right\|_{L^{2}(0,1)} \approx 2.21 \times 10^{-4}$ after 855 iterations.

We also emphasize that we may consider the more realistic situation where null Neumann boundary limit holds on the free part, here $x=0$. It suffices to start with $u^{0} \in \mathscr{A}_{N}=\left\{u \in H^{1}\left(Q_{T}\right), u(\cdot, T)=0, u(\cdot, 0)=u_{0}, u_{x}(0, t)=0\right\}$, and impose that both the descent direction and the corrector have null derivatives at $x=0$. Figure 7 shows the function $u_{h} \in \mathscr{A}_{N}$ associated with $u_{0}(x)=\sin ^{2}(\pi x)$. Figure 8 depicts the corresponding iso-values of $u_{h}$. With $\varepsilon=10^{-5}$, the convergence is reached after 3,431 iterations, and we get $\left\|\bar{u}_{h}(\cdot, T)\right\|_{L^{2}(0,1)} \approx 1.31 \times 10^{-2}$. The convergence is slower in this case because null 

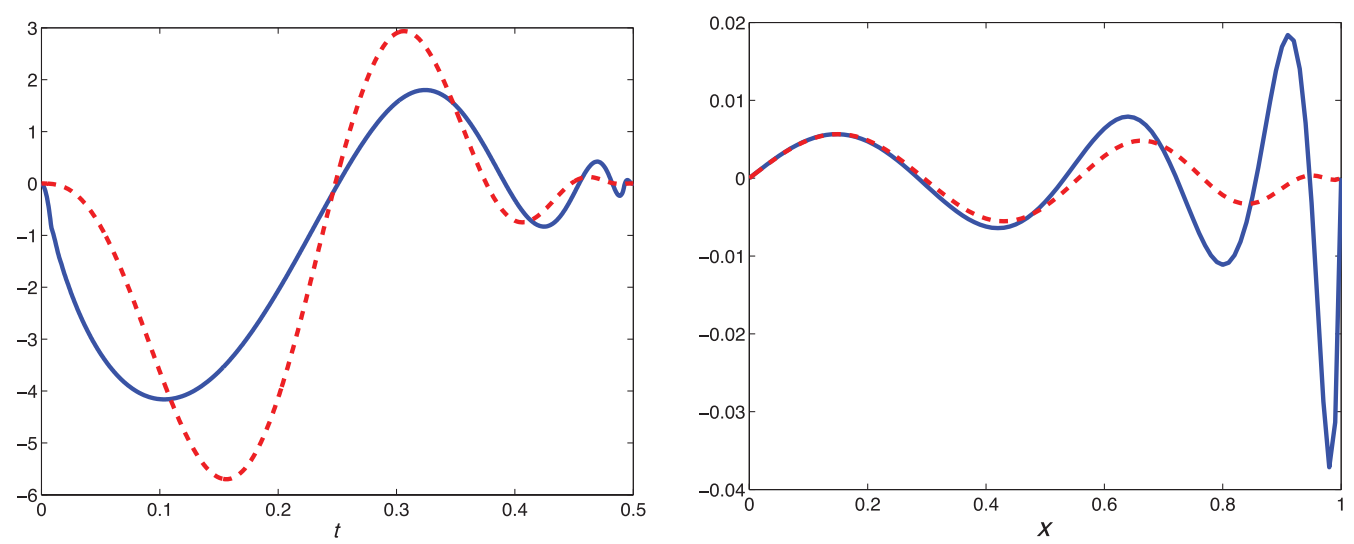

Figure 4. (Colour online) Boundary case $-u_{0}(x)=\sin (\pi x)$. Full line: Trace $u_{h}(x=1, t)$ versus $t \in(0, T)$ (left), and a posteriori solution $\bar{u}_{h}(x, T)$ versus $x \in(0,1)$ (right); dashed line: same quantities obtained with an additional compact support function in time.
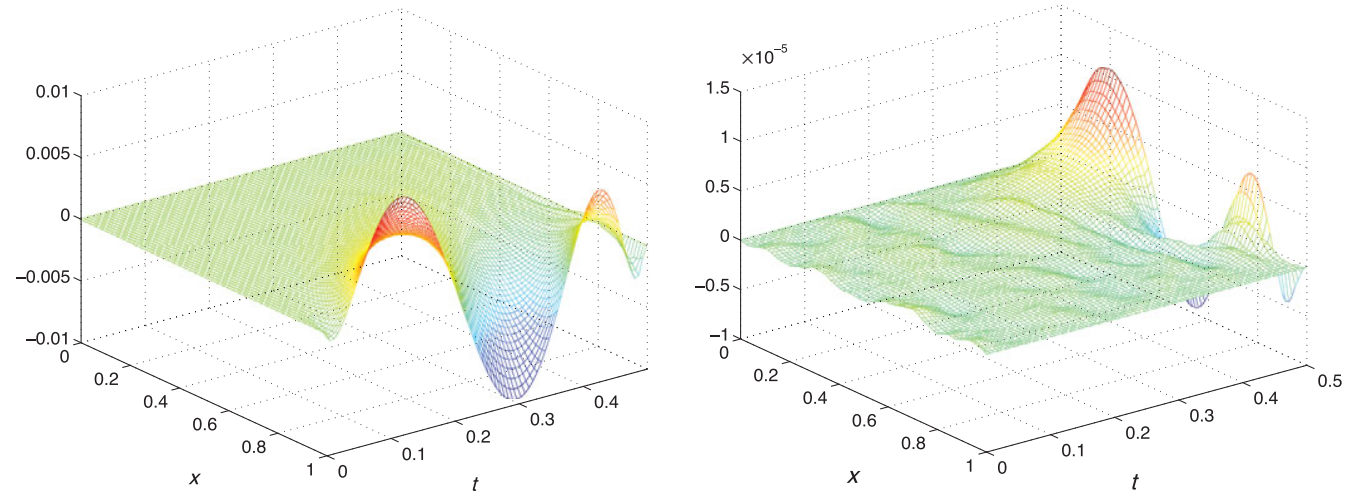

FIGURE 5. (Colour online) Boundary case $-u_{0}=0$. Control acting on $0-\varepsilon=10^{-6}$. Solution in $u_{h} \in \mathscr{A}_{h}$ (left), and the corresponding corrector $v_{h}$ (right) along $Q_{T} . E_{h}\left(u_{h}^{n=1242}\right) \approx 6.63 \times 10^{-9}$ and $\left\|\bar{u}_{h}(\cdot, T)\right\|_{L^{2}(0,1)} \approx 2.89 \times 10^{-5}$.

Neumann boundary condition - contrary to null Dirichlet one - does not emphasize the dissipation of the solution.

Finally, let us comment on a simple way to smooth out the control near $t=T$, and therefore avoid the oscillations that we mentioned at the beginning of this section (see Figure 4 (left)). It suffices to replace at each iteration $n$ the descent direction $g^{n}$ by $c(t) g^{n}$ with any smooth positive function $c$ such that $c(T)=c^{\prime}(T)=0$. Figure 4 gives (in dashed line) the quantities $u_{h}(1, \cdot)$ on $(0, T)$ and $\bar{u}(\cdot, T)$ on $(0,1)$ obtained with $c(t)=\sin ^{2}(\pi t / T)$ (in that case, note that the solution is also smoothed at $t=0$ ). This modification has the effect to reduce the a posteriori error $\left\|\bar{u}_{h}\right\|_{L^{2}(0,1)}$ but to increase the number of iterations. Note also that the $L^{2}$-norm of the trace is larger (see Table 2).

We observe similar results with the Dirichlet boundary condition on $(0,1) \times\{0, T\}$ in (2.2). The CG algorithm converges faster and leads to a control with smaller $L^{2}$-norm. The a posteriori error $\left\|\bar{u}_{h}\right\|_{L^{2}(0,1)}$ is however larger. 
Table 2. Boundary case $-u_{0}(x)=\sin (\pi x)-\varepsilon=10^{-5}$. Numerical results with respect to $h=(\Delta x, \Delta t)$ with a compact support function in time

\begin{tabular}{lcccc}
\hline \hline$\Delta x=\Delta t$ & $1 / 25$ & $1 / 50$ & $1 / 100$ & $1 / 200$ \\
\hline \# CG iteration & 2,552 & 2,724 & 3,689 & 4,276 \\
$\left\|u_{h}\right\|_{L^{2}\left(Q_{T}\right)}$ & $5.19 \times 10^{-1}$ & $5.26 \times 10^{-1}$ & $5.57 \times 10^{-1}$ & $5.71 \times 10^{-1}$ \\
$\left\|u_{h}\right\|_{H^{1}\left(Q_{T}\right)}$ & 7.052 & 7.092 & 7.889 & 8.285 \\
$\left\|u_{h}\right\|_{L^{2}\left(\Sigma_{T}\right)}$ & 1.526 & 1.554 & 1.678 & 1.738 \\
$\left\|\bar{u}_{h}(\cdot, T)\right\|_{L^{2}(0,1)}$ & $9.08 \times 10^{-3}$ & $5.25 \times 10^{-3}$ & $3.46 \times 10^{-3}$ & $2.83 \times 10^{-3}$ \\
$\left\|u_{h}-\bar{u}_{h}\right\|_{L^{2}\left(Q_{T}\right)}$ & $9.51 \times 10^{-3}$ & $2.73 \times 10^{-3}$ & $1.19 \times 10^{-3}$ & $9.61 \times 10^{-4}$ \\
$E_{h}\left(u_{h}\right)$ & $2.88 \times 10^{-6}$ & $2.17 \times 10^{-6}$ & $1.20 \times 10^{-6}$ & $1.19 \times 10^{-6}$ \\
\hline \hline
\end{tabular}
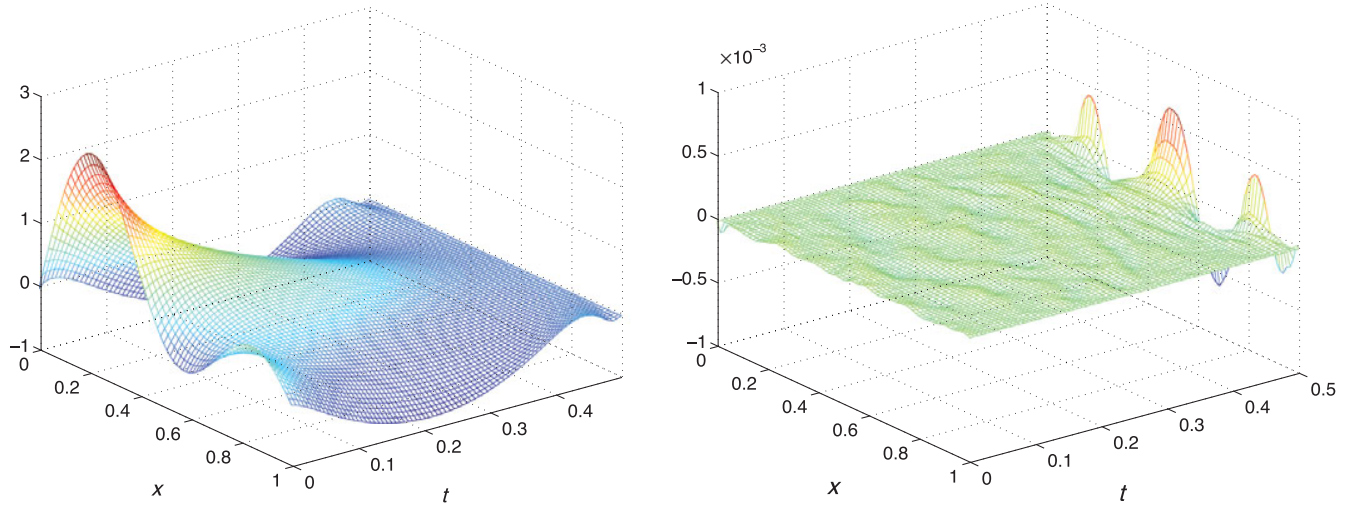

FIGURE 6. (Colour online) Boundary control $-u_{0}(x)=\sin (\pi x)+\sin (2 \pi x)+\sin (3 \pi x)$. Control acting on $\{0,1\}-\varepsilon=10^{-5}$. Solution in $u_{h} \in \mathscr{A}_{h}$ (left), and the corresponding corrector $v_{h}$ (right) along $Q_{T}$.

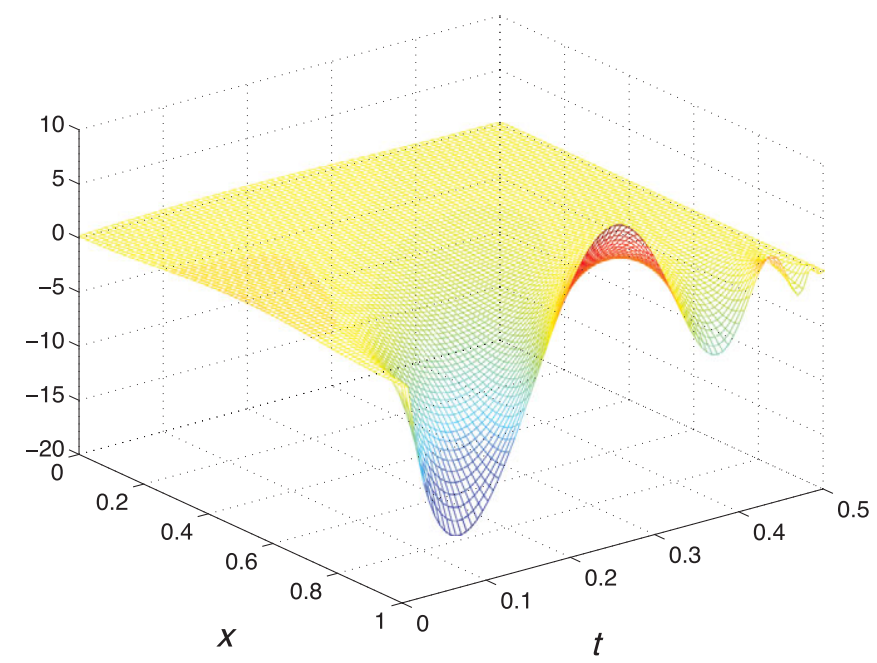

Figure 7. (Colour online) Boundary case $-u_{0}(x)=\sin ^{2}(\pi x)$. Controlled solution $u_{h}$ over $Q_{T}$ with free Neumann boundary condition at $x=0$. 


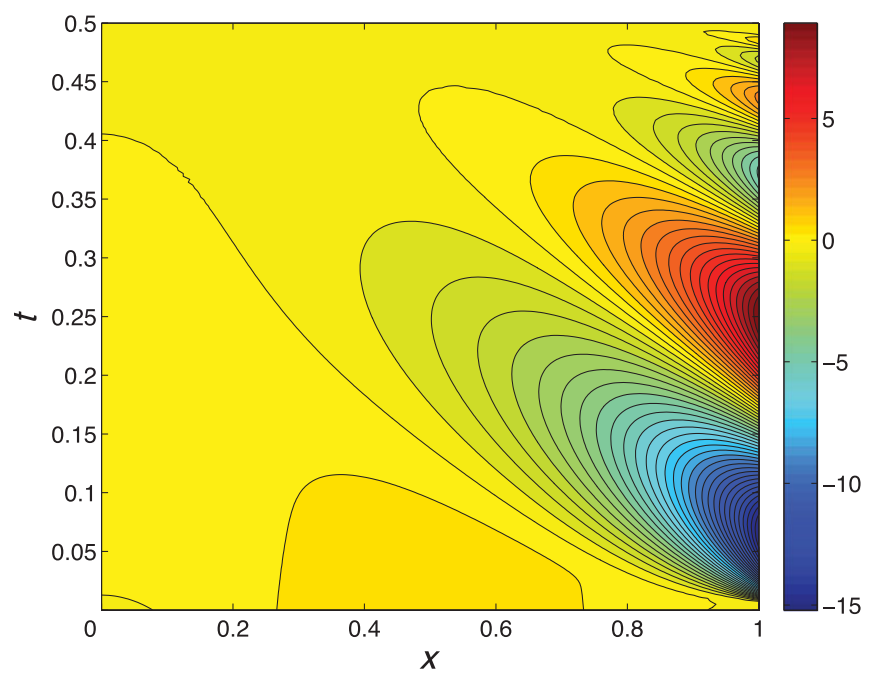

FIGURE 8. (Colour online) Boundary case $-u_{0}(x)=\sin ^{2}(\pi x)$. Iso-values of $u_{h} \in \mathscr{A}_{h}$ along $Q_{T}$ with free Neumann boundary condition at $x=0$.

\subsection{Experiment 2: inner case}

Let us consider the following data: $\omega=(0.2,0.5), T=1 / 2$ and $a(x)=a_{0}=1 / 4$ used notably in $([11,29])$. The initial data to be controlled are again $u_{0}(x)=\sin (\pi x)$.

Table 3 collects some numerical values obtained with the CG algorithm and $\varepsilon=10^{-6}$. In order to have a vanishing control at time $t$, we simply replace in the formulation (2.8) the characteristic function $1_{\omega}(x)$ by $1_{\omega}(x) m(t)$ for any smooth time function $m$ such that $m(T)=0$. The control is thus $f(x, t) 1_{\omega}(x) m(t)$. Here we take $m(t)=(1-t / T)^{2}$. The situation is more favourable than the boundary case in the sense that the number of iterations to reach a relative residual of order $10^{-6}$ (instead of $10^{-5}$ in Table 1) is significantly reduced. The evolution of the cost function is given in Figure 9. As a consequence, the a posteriori error $\left\|\bar{u}_{h}(\cdot, T)\right\|_{L^{2}(0,1)}$ is smaller, of the order $\mathcal{O}\left(10^{-5}\right)$. We also check the boundedness for the $L^{2}\left(q_{T}\right)$ norm of $f_{h} m(t)$ with respect to $h$, and converges as $h \rightarrow 0$. The controlled solution $u_{h} \in \mathscr{A}$ and the corresponding control $f_{h} 1_{\omega}(x) m(t)$ are depicted on Figures 10 and 11 respectively for $(\Delta t, \Delta x)=(1 / 100,1 / 100)$. The isovalues of the corrector function $v_{h}$ are depicted in Figure 12. Note that this control, obtained with the initial guess $u^{0}(x)=\sin (\pi x)(1-t / T)^{2}$, is quite different from the controls obtained by duality arguments in [29]. Mainly concentrated at the beginning of the time interval (it is the effect of the decreasing positive function $m(t)$ ), this $L^{2}$-norm is larger: for $h=(1 / 100,1 / 100)$, we obtain $\left\|f(x, t) 1_{\omega}(x) m(t)\right\|_{L^{2}\left(Q_{T}\right)} \approx 2.839$, about twice the HUM-control obtained in [29].

\section{Remarks on a non-linear situation}

As a good way to emphasize the flexibility of the variational approach to adapt itself to various different settings, we are going to indicate the changes needed for a typical non-linear situation where a low-order non-linear perturbation is considered (see [21]). 
Table 3. Inner case $-u_{0}(x)=\sin (\pi x), T=1 / 2, a_{0}=1 / 4, \Delta x=\Delta t=1 / 100-\omega=(0.2,0.5)$ $-\varepsilon=10^{-6}$. Numerical results with respect to $h=(\Delta x, \Delta t)$ with a compact support function in time

\begin{tabular}{lcccc}
\hline \hline$\Delta x=\Delta t$ & $1 / 25$ & $1 / 50$ & $1 / 100$ & $1 / 200$ \\
\hline \# CG iterates & 135 & 192 & 231 & 361 \\
$\left\|u_{h}\right\|_{L^{2}\left(Q_{T}\right)}$ & $2.53 \times 10^{-1}$ & $2.58 \times 10^{-1}$ & $2.57 \times 10^{-1}$ & $2.61 \times 10^{-1}$ \\
$\left\|u_{h}\right\|_{H^{1}\left(Q_{T}\right)}$ & 1.301 & 1.336 & 1.337 & 1.352 \\
$\left\|f_{h}(x, t) 1_{\omega}(x) m(t)\right\|_{L^{2}\left(Q_{T}\right)}$ & 1.675 & 2.641 & 2.839 & 2.981 \\
$\left\|\bar{u}_{h}(\cdot, T)\right\|_{L^{2}(0,1)}$ & $7.23 \times 10^{-5}$ & $5.43 \times 10^{-5}$ & $4.30 \times 10^{-5}$ & $2.91 \times 10^{-5}$ \\
$\left\|u_{h}-\bar{u}_{h}\right\|_{L^{2}\left(Q_{T}\right)}$ & $3.21 \times 10^{-5}$ & $7.31 \times 10^{-5}$ & $5.10 \times 10^{-5}$ & $1.58 \times 10^{-5}$ \\
$E_{h}\left(u_{h}\right)$ & $4.12 \times 10^{-7}$ & $3.34 \times 10^{-7}$ & $4.16 \times 10^{-7}$ & $2.36 \times 10^{-7}$ \\
\hline \hline
\end{tabular}

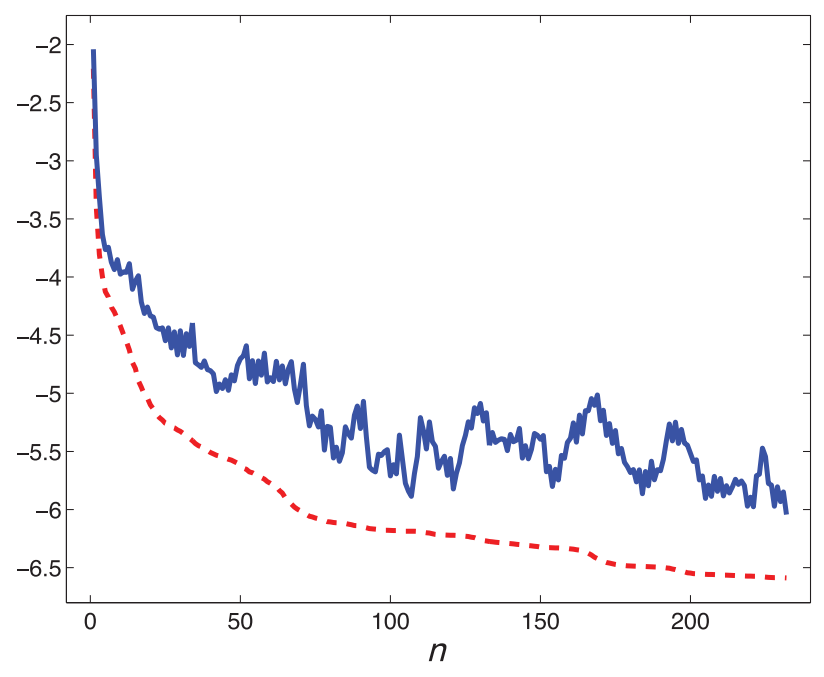

Figure 9. (Colour online) Inner case $-u_{0}(x)=\sin (\pi x)$. Control acting on $\omega=(0.2,0.5)-\varepsilon=10^{-6}$ $-\log _{10}\left(E_{h}\left(u_{h}^{n}\right)\right.$ (dashed line) and $\log _{10}\left(\left\|g_{h}^{n}\right\|_{\mathscr{A}}\right)$ (full line) versus the iteration $n$ of the CG algorithm.

Namely, we will look at the problem of finding a control $w$, so that the solution of the problem

$$
\left\{\begin{array}{lc}
u_{t}-\left(a(x) u_{x}\right)_{x}+F(u)=0, & (x, t) \in Q_{T}, \\
u(x, 0)=u_{0}(x), & x \in(0,1), \\
u(0, t)=0, u(1, t)=w(t), & t \in(0, T)
\end{array}\right.
$$

will comply with $u(x, T)=0$ for all $x \in(0,1)$. System (6.1) is known to be controllable, uniformly with respect to the data $u_{0}$ and $T$ if the nonlinear function $F(s)$ grows slower than $s \log ^{3 / 2}(1+|s|)$ as $|s| \rightarrow+\infty$ (we refer to [13] and also to [6]). Therefore, our approximation scheme can be used in such situations where controllability is known to hold. To our knowledge, the numerical approximation of controls in that nonlinear 


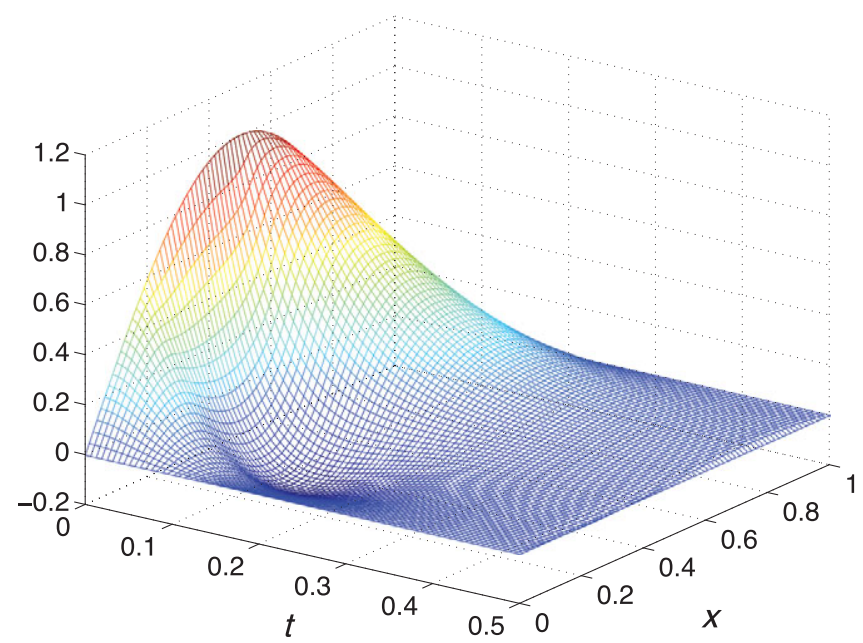

FIgURE 10. (Colour online) Inner case $-u_{0}(x)=\sin (\pi x)$. Solution in $u_{h} \in \mathscr{A}_{h}$ along $Q_{T}$.

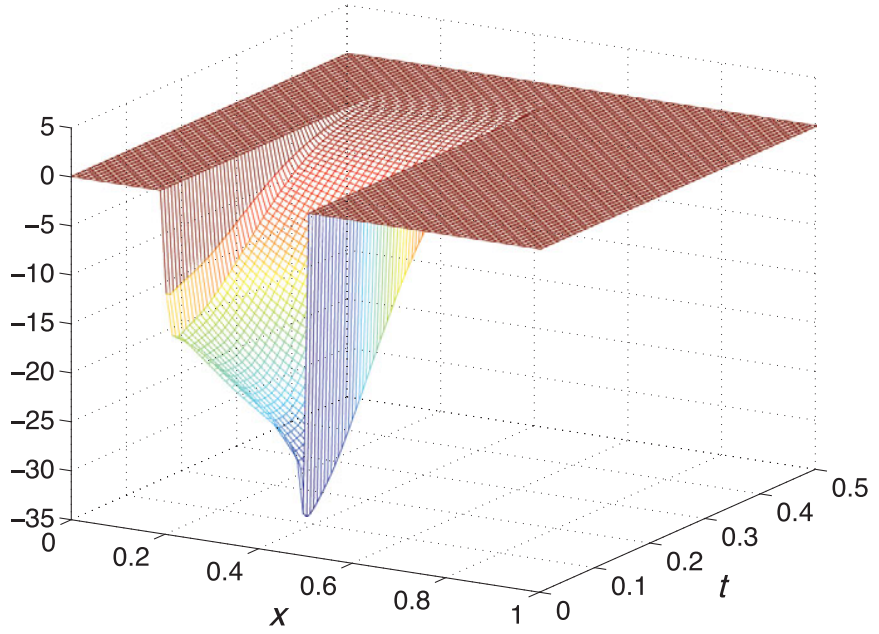

Figure 11. (Colour online) Inner case $-u_{0}(x)=\sin (\pi x)$. Control $f_{h}(x, t) 1_{\omega}(x) m(t)$ along $Q_{T}$.

context has been only addressed recently in $[9,10]$ using linearization and fixed point arguments.

The procedure for such non-linear system is similar. We define the corrector associated with $u$, through the problem

$$
\left\{\begin{array}{lc}
u_{t}-v_{t t}-\left(a(x)\left(u_{x}+v_{x}\right)\right)_{x}+F(u)=0, & (x, t) \in Q_{T}, \\
v_{t}(x, 0)=v_{t}(x, T)=0, & x \in(0,1), \\
v(0, t)=v(1, t)=0, & t \in(0, T),
\end{array}\right.
$$

while the error functional $E$ is still defined by (2.3). Because of controllability, we know that the problem of minimizing the error is well-posed, and so we can go on to explore a 


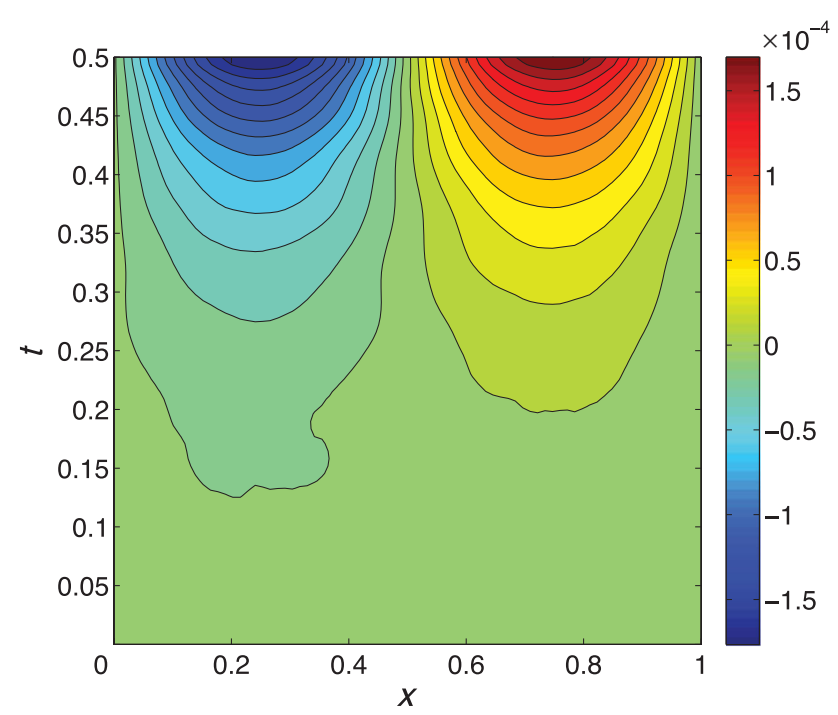

FIGURE 12. (Colour online) Inner case $-u_{0}(x)=\sin (\pi x)$. Iso-values of the corrector $v_{h}$ along $Q_{T}$.

descent procedure for $E$. We obtain the first derivative of $E$ given by

$$
<E^{\prime}(u), U>=-\iint_{Q_{T}}\left(U_{t} v+a(x) U_{x} v_{x}+\left(F^{\prime}(u) \cdot U\right) v\right) d x d t, \quad \forall U \in \mathscr{A}_{0},
$$

leading to the characterization of the corrector $v$ associated with any optimal $u$ (assumed to exist in $\mathscr{A}$ )

$$
\left\{\begin{array}{lc}
v_{t}+\left(a(x) v_{x}\right)_{x}+F^{\prime}(u) v=0, & (x, t) \in Q_{T}, \\
v_{t}(x, 0)=v_{t}(x, T)=0, & x \in(0,1), \\
v(0, t)=v(1, t)=a(1) v_{x}(1, t)=0, & t \in(0, T) .
\end{array}\right.
$$

Once again, the solution of this system vanishes in $Q_{T}$ so that the minimizer of $E$ is a solution of the non-linear heat equation (6.1).

As we have mentioned earlier, even if we are not able to show the well-posedness of the minimization corrector problem, the decrease of the error to zero is a sure indication that the problem is being, at least approximately, controlled. Let us simply mention that, in the conjugate gradient algorithm, the function $g^{n}$ in the steepest descent step is the solution of the linear formulation

$$
\iint_{Q_{T}}\left(g_{t}^{n} \phi_{t}+a(x) g_{x}^{n} \phi_{x}\right) d x d t=-\iint_{Q_{T}}\left(v^{n} \phi_{t}+a(x) v_{x}^{n} \phi_{x}+F^{\prime}\left(u^{n}\right) v^{n} \phi\right) d x d t, \quad \forall \phi \in \mathscr{A}_{0} .
$$

We consider here the function $F$ given by

$$
F(s)=-\alpha s \log ^{p}(1+|s|), \quad \alpha=5, \quad p=1.4 .
$$

Clearly, $F$ belongs to $C^{1}(\mathbb{R})$ and we have $F^{\prime}(s)=-\alpha\left[\log ^{p}(1+|s|)+p \log ^{p-1}(1+|s|)\right)|s| /(1+$ $|s|))$. 


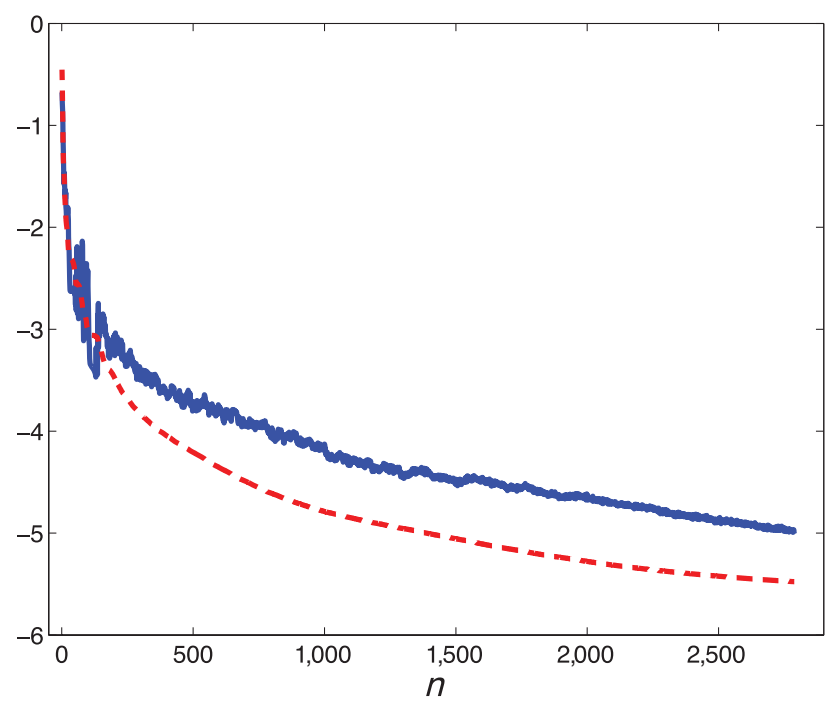

Figure 13. (Colour online) Non-linear case $-u_{0}(x)=3 \sin (\pi x)-F(s)=-5 \operatorname{sog}^{1.4}(1+|s|)-T=1 / 2$, $a_{0}=1 / 4, \Delta x=\Delta t=1 / 100-\log _{10}\left(E_{h}\left(u_{h}^{n}\right)\right)$ (dashed line) and $\log _{10}\left(\left\|g_{h}^{n}\right\|_{\mathscr{A}}\right)$ (full line) versus the iteration $n$ of the CG algorithm.

We take $u_{0}(x)=3 \sin (\pi x)$, the other data are kept unchanged. This non-linear term prevents the diffusion of the heat solution in time, that is, the $L^{2}$-norm $\|u(\cdot, t)\|_{L^{2}(0,1)}$ increases with respect to $t$ : in the uncontrolled situation, we get a norm $\|u(\cdot, T)\|_{L^{2}(0,1)} \geqslant$ $10^{10}$ to be compared with $\|u(\cdot, T)\|_{L^{2}(0,1)} \approx 2.05 \times 10^{-1}$ for the linear case. The situation is therefore much more difficult than the linear one. However, our approach allows to drive the solution in a closed neighbourhood of zero: the a posteriori error that we get is $\left\|\bar{u}_{h}(\cdot, T)\right\|_{L^{2}(0,1)} \approx 1.92 \times 10^{-3}$. Figure 13 depicts the evolution of $E_{h}\left(u_{h}^{n}\right)$ and the residual $\left\|g_{h}^{n}\right\|_{\mathscr{A}}$ (in $\log _{10}$ scale) with respect to the iteration of the algorithm.

The control that we obtain is still oscillating along the time and has a much larger amplitude (compared with the linear situation), specially near $t=0$, so as to avoid the blow up of the solution (see Figure 14 (left)). Note that we have used the compact support function $c(t)=\sin ^{2}(\pi t / T)$ so that $u_{h}$ is smooth near $T$. The non-linearity increases slightly the number of iterations, here 2,788 , to reach the same threshold, $\varepsilon=10^{-5}$. We also plot the corrector $v_{h}$ in $Q_{T}$ (see Figure 14 (right)) and the iso-values of the solution $u_{h}$ (see Figure 15). For larger values of $\alpha$, the algorithm does not converge anymore. Similar phenomena are observed for smaller larger values of $\left\|y_{0}\right\|_{L^{\infty}(0,1)}$. We also obtain convergence results for the case $F(s)=-5|s| \log (1+|s|)$, more critical than the previous situation since $f$ is non-positive. The number of iterations is greater $(6,883)$ as well as the $L^{2}\left(\Sigma_{T}\right)$-norm of the control. Similar remarks hold for 'more' non-linear function such as $F(s)=\alpha|s|^{p}, p \in \mathbb{N}$ or $F(s)=\alpha \exp (s)$, provided that $\alpha$ or $\left\|y_{0}\right\|_{L^{2}(0,1)}$ be small enough.

\section{Reducing the norm of the control}

By minimizing the error functional $E$ defined by (2.3), we do not control any norm, in particular the $L^{2}$-norm, of the trace of the solution on $\Sigma_{T}$. From the practical viewpoint, 

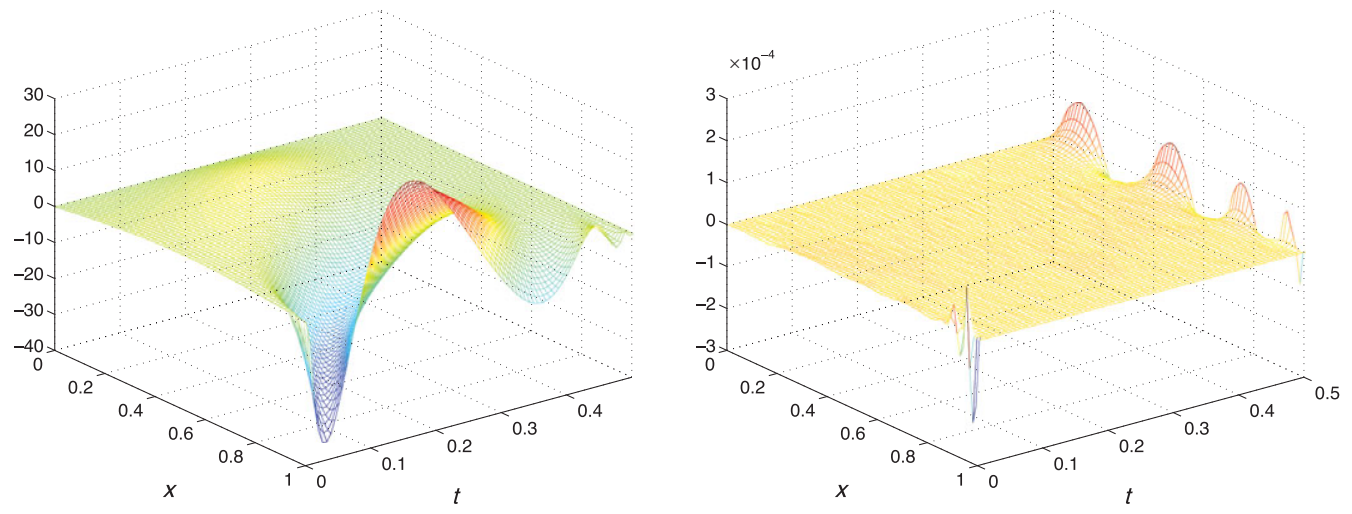

FIgURE 14. (Colour online) Non-linear case $-u_{0}(x)=3 \sin (\pi x)$. Control acting on $x=0-$ $\varepsilon=10^{-5}$. Solution in $u_{h} \in \mathscr{A}_{h}$ (left) and corrector $v_{h}$ (right) along $Q_{T} . E_{h}\left(u_{h}^{n=2788}\right) \approx 3.33 \times 10^{-6}$, $\left\|g_{h}^{n=2788}\right\|_{\mathscr{A}} \approx 9.89 \times 10^{-6}$ and $\left\|\bar{u}_{h}(\cdot, T)\right\|_{L^{2}(0,1)} \approx 1.92 \times 10^{-3}$.

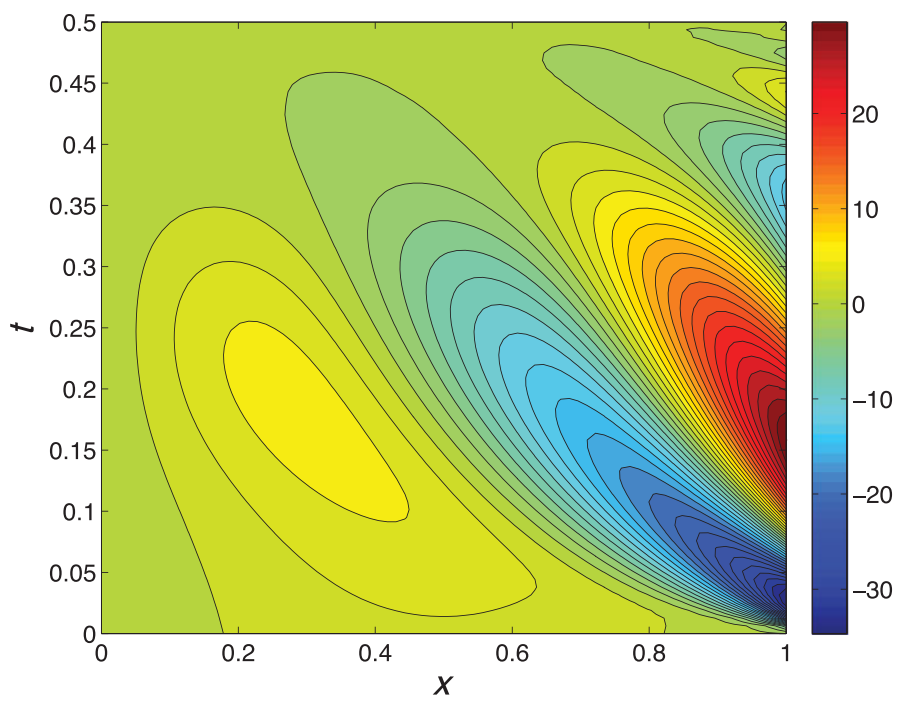

FIGURE 15. (Colour online) Non-linear case $-u_{0}(x)=3 \sin (\pi x)$. Control acting on $x=0-$ $\varepsilon=10^{-5}$ - iso-values of $u_{h} \in \mathscr{A}_{h}$ along $Q_{T}$.

it is interesting to minimize such norm. A possibility is to take advantage of the fact that the method allows to obtain non-trivial controlled solutions in $\mathscr{A}$ with null initial condition $u_{0}$, that is, solutions in $\mathscr{A}_{0}$. Suppose a family $\left\{u_{k}\right\}_{k \in[1, N]}$ of $N$ elements in $\mathscr{A}_{0}$ is given. Then for any $\alpha_{n} \in \mathbb{R}, n=1 \cdots N$ and any $u \in \mathscr{A}$,

$$
u^{N}(x, t)=u(x, t)+\sum_{k=1}^{N} \alpha_{k} u_{k}(x, t), \quad(x, t) \in Q_{T}
$$

still belongs, in the linear situation of Section 2 , to $\mathscr{A}$. The minimization of $\left\|u^{N}(1, t)\right\|_{L^{2}(0, T)}$ is then reduced to a quadratic minimization on $\left\{\alpha_{k}\right\}_{k=1, N}$. The method that we propose to 


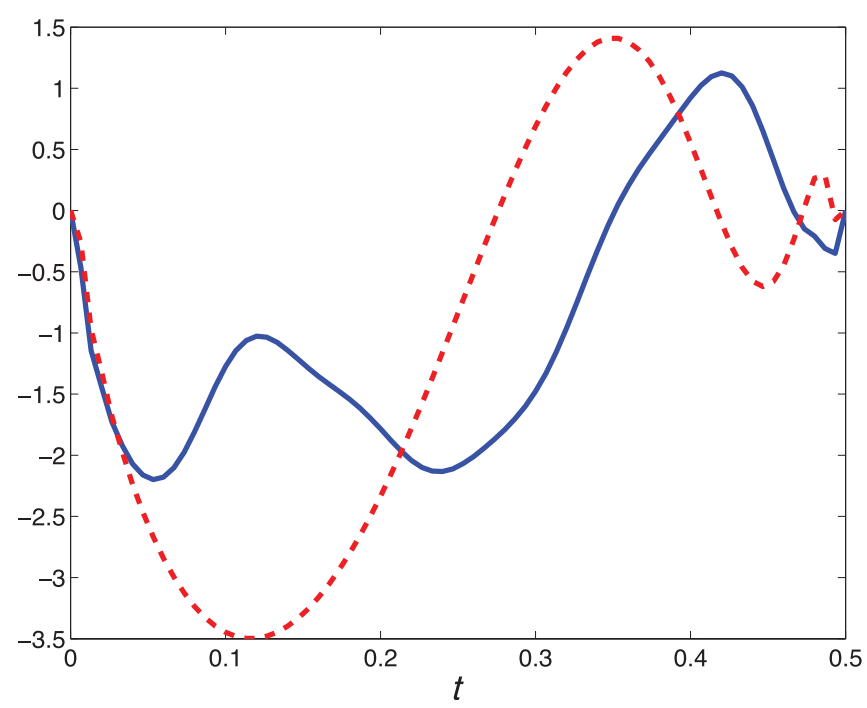

Figure 16. (Colour online) $u_{0}(x)=\sin (\pi x)-T=1 / 2, a_{0}=1 / 4, \Delta x=\Delta t=1 / 100$. Trace of $u_{h}^{N}$ (full line) and of $u_{h}$ (dashed line) along $\Sigma_{T}$.

construct the family $\left\{u_{k}\right\}_{k \in[1, N]}$ is as follows: We first compute $N$ elements $v_{k}, k=1, \cdots, N$ in $\mathscr{A}_{0}$ using the conjugate gradient algorithm with initial guesses $u_{k}^{0}(x, t)=x \sin (k \pi t / T)^{2}$. Then we orthogonalize these elements using the Gram-Schmidt procedure with the scalar product associated with $\mathscr{A}$ :

$$
u_{k}=v_{k}-\sum_{n=1}^{k-1}<v_{k}, u_{n}>_{\mathscr{A}} u_{n} .
$$

Figure 16 shows the trace of $u^{N} \in \mathscr{A}$ along $\Sigma_{T}$ obtained with $N=10$ as well as the trace of $u \in \mathscr{A}$ corresponding to $u^{0}(x)=\sin (\pi x)(1-t / T)^{2}$ (see Figure 2). We obtain $\left\|u_{h}^{N}\right\|_{L^{2}\left(\Sigma_{T}\right)} \approx 0.981$ lower than $\left\|u_{h}\right\|_{L^{2}\left(\Sigma_{T}\right)} \approx 1.392$. Larger values of $N$, which require a finer mesh in time so as to capture the oscillating functions $\sin (m \pi t / T)$, do not allow a significant additional reduction of the $L^{2}\left(\Sigma_{T}\right)$-norm.

This constructive approach that allows to jump from a local minimum of $E$ to another one does not apply for the non-linear situation given in Section 6. On the other hand, the more flexible approach that consists to minimize at the same time the error functional $E$ and the $L^{2}$-norm of the trace with respect to $u$ does not lead to satisfactory results, as it depends too much on the initial guess $u^{0}$. In that respect, a possible strategy could be to initialize the $\mathrm{CG}$ algorithm with an approximate control obtained from the dual approach (see [4]). Nevertheless, since the set $\{u \in \mathscr{A}, E(u)=0\}$ is convex, we may apply the Uzawa-type method (see, for instance, [15]) and minimize over $\mathscr{A} \times \mathbb{R}$ the Lagrangian

$$
L(u, \lambda)=\frac{1}{2}\|u(1, t)\|_{L^{2}(0, T)}^{2}+\lambda E(u) .
$$

The real $\lambda$ is thus the multiplier corresponding to the constraint $E(u)=0$. Starting from 
any $\lambda^{0} \in \mathbb{R}$, the algorithm aims to define a sequence of pair $\left(u^{k}, \lambda^{k}\right) \in \mathscr{A} \times \mathbb{R}, k \geqslant 0$ according to the following two steps:

- Compute $u^{k}$ such that $L\left(u^{k}, \lambda^{k}\right) \leqslant L\left(v, \lambda^{k}\right), \quad \forall v \in \mathscr{A}$.

- Compute $\lambda^{k+1}:=\lambda^{k}+\rho E\left(u^{k}\right), \quad \rho>0$.

The first step is performed using the gradient method developed in Sections 2 and 4, the functional $E$ being replaced by the functional $L\left(\cdot, \lambda^{k}\right)$. First experiments lead to satisfactory results and will be given in a distinct work.

\section{Concluding remarks}

The variational approach that we discussed here to construct numerical controls is very different in nature from the usual one [4,29] which makes use of dual variable to deal with the constraint $u(\cdot, T)=0$. In the context of parabolic equations, this difference is significant because the variational approach avoids the approximation of singular functional spaces and therefore ill-posed problems. Here the problem is elliptic and leads to standard and well-posed formulations. A quantitative comparison with the dual approach for the boundary situation remains however to be done.

The method extends to any target - trajectory for the heat equation - to higher dimensions, and to any system for which a controllability result is known. In particular, we may consider the heat equation with zero-order term and address - as we have written in Section 6 - the controllability of semi-linear heat equations in a different form compared with linearization and fixed point arguments. It has been also used successfully for the Stokes problem [28].

It is also remarkable to note that this variational approach allows to solve inverse problems. Let us mention, in particular, the highly ill-posed backward heat problem which consists to determine the solution of the heat equation at time $t=0$ from the solution $u_{T}$ at any positive time $T$ (we refer to [17]). It suffices to define the functional spaces $\mathscr{A}$ and $\mathscr{A}_{0}$ respectively as follows : $\mathscr{A}=\left\{u \in H^{1}\left(Q_{T}\right), u(0, t)=u(1, t)=0, u(x, T)=u_{T}(x),(x, t) \in\right.$ $\left.Q_{T}\right\}$ and $\mathscr{A}_{0}=\left\{u \in H^{1}\left(Q_{T}\right), u(0, t)=u(1, t)=0, u(x, T)=0,(x, t) \in Q_{T}\right\}$. We plan to analyse this situation in the future.

\section{Acknowledgements}

This research is supported in part by MTM2007-62945 of the MCyT (Spain), and PCI080084-0424 of the JCCM (Castilla-La Mancha).

\section{References}

[1] Alessandrini, G. \& Escauriaza, L. (2008) Null-controllability of one-dimensional parabolic equations. ESAIM Control Optim. Calc. Var. 14(2), 284-293.

[2] Ben Belgacem, F. \& Kaber, S. M. (2011) On the Dirichlet boundary controllability of the 1-D heat equation: Semi-analytical calculations and ill-posedness degre. Inverse Probl. 27(5), 055012, 19 pp.

[3] Boyer, F., Hubert, F. \& Le Rousseau, J. (2011) Uniform null-controllability properties for space/time-discretized parabolic equations. Numer. Math. 118(4), 601-661. 
[4] Carthel, C., Glowinski, R. \& Lions, J.-L. (1994) On exact and approximate boundary controllability for the heat equation: A numerical approach. J. Optim. Theory Appl. 82(3), 429-484.

[5] Conon, J. M. (2007) Control and Nonlinearity, AMS Mathematical Surveys and Monographs, Vol. 136, American Mathematical Society, Providence, RI.

[6] Coron, J. M. \& TrÉlat, E. (2004) Global steady-state controllability of one-dimensional semi-linear heat equations. SIAM J. Control Optim. 43, 549-569.

[7] Ervedoza, S. \& Valein, J. (2010) On the observability of abstract time-discrete linear parabolic equations. Rev. Mat. Comput. 23(1), 163-190.

[8] Fattorini, H. O. \& Russel, D. L. (1971) Exact controllability theorems for linear parabolic equation in one space dimension. Arch. Ration. Mech. 43, 272-292.

[9] Fernández-CARA, E. \& MüNCH, A. (2011) Numerical null controllability of a semi-linear 1D heat equation via a least squares reformulation. C.R. Acad. Sci. Paris Sér. I 349, 867-871.

[10] Fernández-Cara, E. \& MünCh, A. (2012) Numerical null controllability of semi-linear 1D heat equations: Fixed points, least squares and Newton methods. Math. Control Relat. Fields 2(3), 217-246.

[11] FernándeZ-CARA, E. \& MÜNCH, A. (2013) Numerical null controllability of the 1D heat equation: Primal algorithms. Séma J. 61(1), 49-78.

[12] FernándeZ-CARA, E. \& MÜNCH, A. (to appear) Numerical null controllability of the 1D heat equation: Carleman weights and duality. J. Opt. Th. Appl. doi: 10.1007/s10957-013-0517-z.

[13] FernándeZ-CARA, E. \& Zuazua, E. (2000) Null and approximate controllability for weakly blowing up semilinear heat equations Ann. Inst. H. Poincaré Anal. Non Linéaire 17(5), 583616.

[14] Fursikov, A. V. \& Yu, O. Imanuvilov (1996) Controllability of Evolution Equations, Lecture Notes Series, No. 34. Seoul National University, Korea, 163 pp.

[15] Glowinski, R. (1983) Numerical Methods for Nonlinear Variational Problems, Springer series in Computational Physics, Springer, New York, NY.

[16] Glowinski, R., Lions, J. L. \& He, J. (2008) Exact and Approximate Controllability for Distributed Parameter Systems: A Numerical Approach, Encyclopedia of Mathematics and its Applications, 117. Cambridge University Press, Cambridge, UK.

[17] Hào, D. N. (1998) Methods for Inverse Heat Conduction Problems, Methods and Procedures in Mathematical Physics, 43, Peter Lang, Frankfurt am Main, Germany.

[18] Kindermann, S. (1999) Convergence rates of the Hilbert Uniqueness Method via Tikhonov regularization. J. Optim. Theory Appl. 103(3), 657-673.

[19] LabBÉ, S. \& TRÉLAT, E. (2006) Uniform controllability of semi-discrete approximations of parabolic control systems, Syst. Control Lett. 55, 597-609.

[20] Laroche, B., Martin, P. \& Rouchon, P. (2000) Motion planning for the heat equation. Int. J. Robust Nonlinear Control 10, 629-643.

[21] Lasiecka, I. \& TRiggiani, R. (1991) Exact controllability of semilinear abstract systems with applications to waves and plates boundary control. Appl. Math. Optim. 23, 109154.

[22] Lasiecka, I. \& Triggiani, R. (2000) Control Theory for Partial Differential Equations: Continuous and Approximation Theories. I. Abstract Parabolic Systems. Encyclopedia of Mathematics and its Applications, 74, Cambridge University Press, Cambridge, UK.

[23] Lebeau, G. \& Robbiano, L. (1995) Contrôle exact de l'équation de la chaleur. Comm. Partial Differ. Equ. 20(1-2), 335-356.

[24] Lions, J. L. (1971) Optimal Control of Systems Governed by Partial Differential Equations, Springer, New York, NY.

[25] Lions, J. L. (1988) Exact controllability, stabilizability and perturbations for distributed systems. SIAM Rev. 30, 1-68.

[26] Micu, S. \& Zuazua, E. (2011) On the regularity of null-controls of the linear 1-D heat equation. C. R. Acad. Sci. Paris, Ser. I 349, 673-677. 
[27] Micu, S. \& Zuazua, E. (2011) Regularity issues for the null-controllability of the linear 1-D heat equation. Syst. Cont. Lett. 60(6), 406-413.

[28] MÜNCh, A. \& Pedregal, P. (2013) A least-squares formulation for the approximation of null controls for the Stokes system. C. R. Acad. Sci. Paris, Ser. I 351, 545-550.

[29] MüNCH, A. \& ZuAZUA, E. (2010) Numerical approximation of null controls for the heat equation: Ill-posedness and remedies. Inverse Probl. 26(8), 085018, 39pp.

[30] Pedregal, P. (2010) A variational perspective on controllability. Inverse Probl. 26(1), 015004, $17 \mathrm{pp}$.

[31] Pedregal, P. (to appear) Erratum: "A variational perspective on controllability." [Inverse Probl. 26(1), 015004].

[32] Russell, D. L. (1978) Controllability and stabilizability theory for linear partial differential equations. Recent progress and open questions. SIAM Rev. 20, 639-739. 